\title{
Natural antisense transcript of Period2, Per2AS, regulates the amplitude of the mouse circadian clock
}

\author{
Rebecca A. Mosig, ${ }^{1,2}$ Allison N. Castaneda, ${ }^{1,2}$ Jacob C. Deslauriers ${ }_{1}^{1,2}$ Landon P. Frazier, ${ }^{1,2}$ \\ Kevin L. He, ${ }^{1,2}$ Naseem Maghzian, ${ }^{1,2}$ Aarati Pokharel, ${ }^{1,2}$ Camille T. Schrier, ${ }^{1,2,3}$ Lily Zhu, ${ }^{1,2}$ \\ Nobuya Koike, ${ }^{4}$ John J. Tyson, ${ }^{1,2,3}$ Carla B. Green, ${ }^{5}$ Joseph S. Takahashi, ${ }^{5,6}$ and Shihoko Kojima ${ }^{1,2,3}$ \\ ${ }^{1}$ Department of Biological Sciences, Virginia Polytechnic Institute and State University, Blacksburg, Virginia 24061, USA; ${ }^{2}$ Fralin \\ Life Sciences Institute, Virginia Polytechnic Institute and State University, Blacksburg, Virginia 24061, USA; ${ }^{3}$ Division of Systems \\ Biology, Academy of Integrated Science, Virginia Polytechnic Institute and State University, Blacksburg, Virginia 24061; \\ ${ }^{4}$ Department of Physiology and Systems Bioscience, Kyoto Prefectural University of Medicine, Kyoto 062-8566, Japan; \\ ${ }^{5}$ Department of Neuroscience, The University of Texas Southwestern Medical Center, Dallas Texas 75390, USA; ${ }^{6}$ Howard Hughes \\ Medical Institute, The University of Texas Southwestern Medical Center, Dallas, Texas 75390, USA
}

In mammals, a set of core clock genes form transcription-translation feedback loops to generate circadian oscillations. We and others recently identified a novel transcript at the Period2 (Per2) locus that is transcribed from the antisense strand of Per2. This transcript, Per2AS, is expressed rhythmically and antiphasic to Per2 mRNA, leading to our hypothesis that Per2AS and Per2 mutually inhibit each other's expression and form a double negative feedback loop. By perturbing the expression of Per2AS, we found that Per2AS transcription, but not transcript, represses Per2. However, Per2 does not repress Per2AS, as Per2 knockdown led to a decrease in the Per2AS level, indicating that Per2AS forms a single negative feedback loop with Per2 and maintains the level of Per2 within the oscillatory range. Per2AS also regulates the amplitude of the circadian clock, and this function cannot be solely explained through its interaction with Per2, as Per2 knockdown does not recapitulate the phenotypes of Per2AS perturbation. Overall, our data indicate that Per2AS is an important regulatory molecule in the mammalian circadian clock machinery. Our work also supports the idea that antisense transcripts of core clock genes constitute a common feature of circadian clocks, as they are found in other organisms.

[Keywords: Period2; amplitude; antisense transcript; circadian; long noncoding RNA]

Supplemental material is available for this article.

Received August 20, 2020; revised version accepted April 26, 2021.

Eukaryotic genomes are pervasively transcribed, and nonprotein-coding portions of the genome dominate the transcriptional output of mammals (Bertone et al. 2004; Røsok and Sioud 2004; Carninci et al. 2005; Katayama et al. 2005; Sun et al. 2006). RNA species beyond mRNA are known as noncoding RNAs (ncRNAs) and have been categorized into many subtypes, such as ribosomal RNA (rRNA), transfer RNA (tRNA), small nucleolar RNAs (snoRNAs), small nuclear RNAs (snRNAs), microRNAs (miRNAs), circular RNAs (circRNAs), and long noncoding RNAs (lncRNAs) (for review, see Mercer et al. 2009; Panda et al. 2017). Despite their pervasive transcription, ncRNAs, particularly lncRNAs, were originally considered to be mere transcriptional noise and to lack defined functions. Poor evolutionary conservation in their primary sequences between species also raised concerns about

Corresponding author: skojima@vt.edu

Article published online ahead of print. Article and publication date are online at http://www.genesdev.org/cgi/doi/10.1101/gad.343541.120. their functional significance (Ponjavic et al. 2007; Guttman et al. 2009; Johnsson et al. 2014). More recent studies, however, have revealed an intriguing conservation of the genomic positions of lncRNAs (i.e., synteny) as well as conservation of their promoter and exon sequences, compared with intron or nontranscribed intergenic regions of protein-coding genes (Khaitovich et al. 2006; Pang et al. 2006; Yassour et al. 2010; Rhind et al. 2011; Derrien et al. 2012; Goodman et al. 2013; Anderson et al. 2016; Engreitz et al. 2016; Groff et al. 2016). These observations raise the possibility that some lncRNAs, if not all, are biologically relevant and have important and conserved functions. Indeed, a few dozen examples have highlighted the importance of lncRNAs in a variety of

(C) 2021 Mosig et al. This article is distributed exclusively by Cold Spring Harbor Laboratory Press for the first six months after the full-issue publication date (see http://genesdev.cshlp.org/site/misc/terms.xhtml). After six months, it is available under a Creative Commons License (Attribution-NonCommercial 4.0 International), as described at http://creativecommons.org/licenses/by-nc/4.0/. 
biological processes, such as $\mathrm{X}$ chromosome inactivation, imprinting, cell cycle regulation, and stem cell differentiation (Lee et al. 1999; Smilinich et al. 1999; Sleutels et al. 2002; Feng et al. 2006; Dinger et al. 2008; Morris et al. 2008; Zhao et al. 2008; Bond et al. 2009; Sopher et al. 2011; Modarresi et al. 2012).

Circadian rhythmicity, which modulates daily biochemical, physiological, and behavioral cycles, is a fundamental aspect of life on Earth. In mammals, essentially every cell is capable of generating circadian rhythms, and, within each cell, a set of clock genes forms a network of transcription-translation feedback loops that drive oscillations of $\sim 24 \mathrm{~h}$ (for review, see Lowrey and Takahashi 2004; Takahashi et al. 2008; Takahashi 2017). In one of these loops, the heterodimeric transcription activators (BMAL1/CLOCK and its paralog BMAL1/NPAS2) activate transcription of the Period (Per) 1-3 and Cryptochrome (Cry) 1-2 genes, resulting in high levels of these transcripts. The resulting PER and CRY proteins then heterodimerize in the cytoplasm, translocate back to the nucleus, and interact with CLOCK/BMAL1 to inhibit transcription of Per and Cry genes. Subsequently, the PER/CRY repressor complex is degraded, and BMAL1/ CLOCK can now activate a new cycle of transcription. In a second feedback loop, BMAL1/CLOCK activates the expression of orphan nuclear receptor genes $R e v$-erb $\alpha / \beta$ (Nr1d1/2). REV-ERB proteins in turn repress the expression of Bmal1, Clock, Npas2, Cry1, and Nfil3. ROR proteins, also orphan nuclear receptors, recognize the same DNA motif as REV-ERB proteins, compete with their binding, and activate the expression of the same target genes. In the last loop, BMAL1/CLOCK activates the expression of $D b p$, while REV/ROR proteins activate and inhibit the expression of $N f i l 3$, respectively. Both DBP and NFIL3 are transcription factors: DBP activates while NFIL3 represses the transcription of target genes, such as Rev-erbs, Rors, and Pers. These feedback loops constitute the molecular mechanism, as currently understood, of circadian rhythms in mammals (for review, see Takahashi 2017).

Several circadian transcriptome studies discovered a new RNA molecule, which we named Per2AS, that is transcribed within the Per2 locus in mouse liver, lung, kidney, and adrenal gland (Koike et al. 2012; Menet et al. 2012; Vollmers et al. 2012; Fang et al. 2014; Zhang et al. 2014). In mammals, $25 \%-40 \%$ of protein-coding genes have antisense transcript partners (Røsok and Sioud 2004; Katayama et al. 2005; Sun et al. 2006), and some antisense transcripts have been shown to exert functions in a variety of processes, such as cell cycle regulation, genome imprinting, immune response, neuronal function, and cardiac function (Faghihi and Wahlestedt 2009; Khorkova et al. 2014; Wanowska et al. 2018). However, the physiological roles of most antisense transcripts remain uncertain.

In an earlier study, we mathematically tested the hypothesis that Per2AS and Per2 mutually inhibit each other's expression and form a double negative feedback loop, considering three potential mechanisms to describe the Per2AS-Per2 interaction: transcriptional (i.e., transcriptional interference in cis), post-transcriptional (i.e., degra- dation of RNA duplexes in trans), or a combination of both effects. The model predicted that all three mechanisms are consistent with the basic molecular details of circadian rhythms in mouse but that the transcriptional model would lead to a more robust oscillation compared with the post-transcriptional model (Battogtokh et al. 2018).

In this study, we experimentally tested the predictions of these models by perturbing Per2AS expression both transcriptionally and post-transcriptionally. We found that Per2AS transcriptionally represses Per2, indicating that one of the functions of Per2AS is to maintain the level of Per2 within an oscillatory range. This is critical, as Per2 is the only core clock gene for which the abundance, rhythmicity, and the phase of its expression are critical to maintain circadian rhythmicity in mouse (Chen et al. 2009). We also found that Per2AS regulates the amplitude of the circadian clock, and this function does not rely solely on its interaction with Per2. Little is known about the molecular basis of circadian amplitude regulation, although the second feedback loop consisting of Bmall, Clock, Rev-erb $\alpha / \beta$, Ror genes has been implicated to play a central role (Vitaterna et al. 2006; He et al. 2016; Zhao et al. 2016; Littleton et al. 2020). As we establish Per2AS as a new player in amplitude regulation, functional analysis of Per2AS will help us deepen our understanding of the regulatory mechanisms of circadian amplitude. Overall, we propose that Per2AS serves as an important regulatory molecule in the mammalian circadian clock machinery.

\section{Results}

\section{Characterization of Per2AS}

Recent circadian transcriptome studies have identified a novel transcript, Per2AS, at the Per2 locus that is transcribed from the opposite strand and expressed antiphasic to the sense Per2 transcript in mouse liver (Koike et al. 2012; Menet et al. 2012; Vollmers et al. 2012; Fang et al. 2014; Zhang et al. 2014). Our quantitative PCR (qPCR) analyses demonstrated that the Per2AS is indeed expressed rhythmically and antiphasic to Per2 mRNA in mouse liver, and it peaks at ZT 4 (Zeitgeber time, where ZT 0 and ZT 12 are defined as time of lights on and lights off, respectively) (Fig. 1A). Rhythmic and antiphasic expression patterns of Per2AS and Per2 have been observed as well in NIH3T3 cells (Bmal1-luc) and mouse embryonic fibroblasts (MEFs) derived from PER2::LUCIFERASE knock-in mouse (Fig. 1A; Yoo et al. 2004).

Per2AS is spliced and polyadenylated, similar to mRNAs (Fig. 1B; Supplemental Fig. S1A). Rapid Amplification of cDNA Ends (RACE) analysis revealed that the transcription start site (TSS) of Per2AS is located in intron 6 of Per2 (Fig. 1B), which is consistent with the most $5^{\prime}$ signals of Per2AS (Supplemental Fig. S1A; Koike et al. 2012; Menet et al. 2012; Zhang et al. 2014). Strong and rhythmic recruitment of RNAPII-Ser5P (RNA polymerase II whose serine 5 in the C-terminal domain is phosphorylated) has been observed just upstream of Per2AS TSS, indicating an active and rhythmic initiation of transcription at this site 


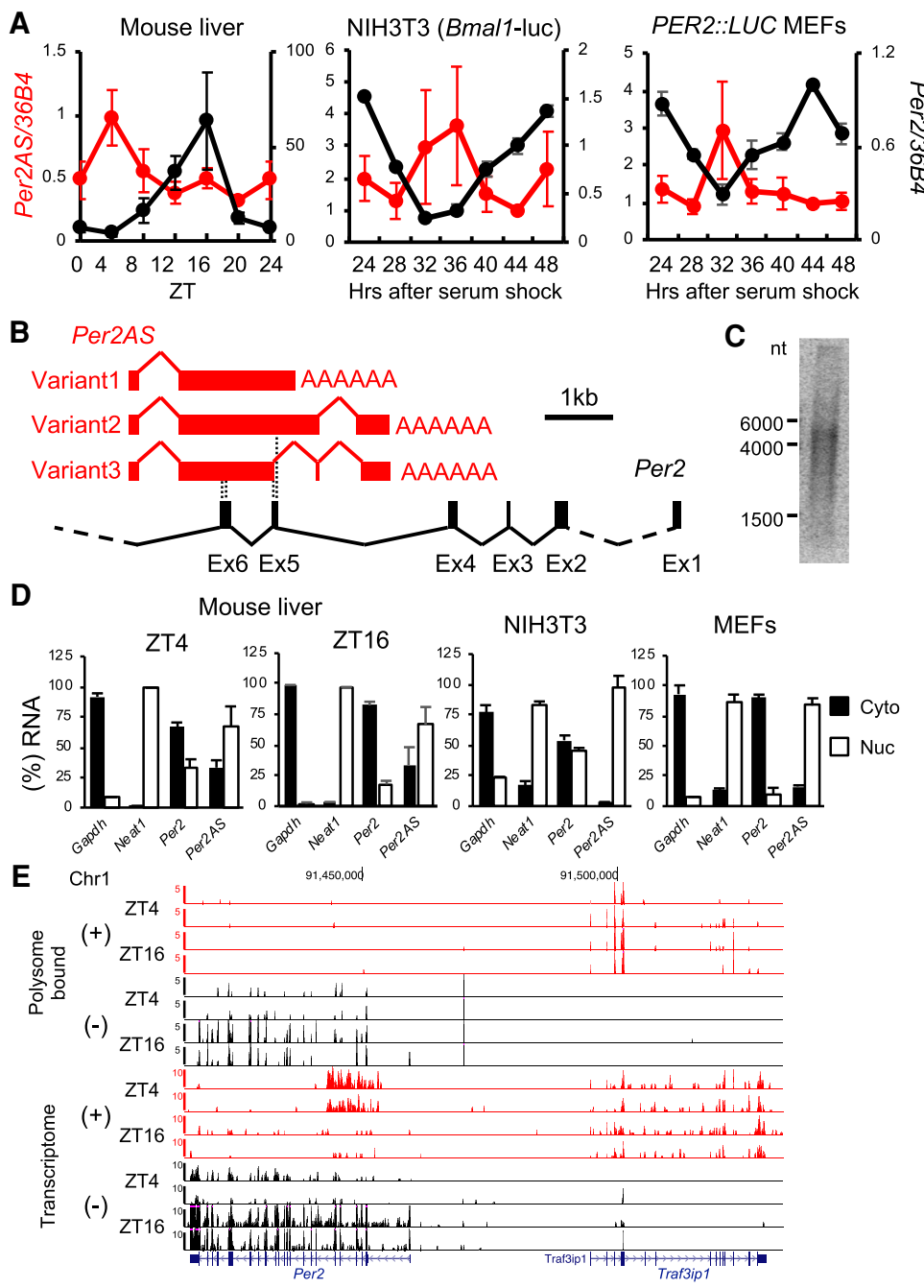

Figure 1. Characterization of Per2AS. (A) Relative RNA expression of Per2AS (red) and Per2 (black) in mouse liver, Bmal1-luc (NIH3T3), and PER2::LUC MEFs. The expression levels of Per2AS at $44 \mathrm{~h}$ after serum shock were set to 1 in Bmal1-luc and PER2::LUC MEFs. (B) Genomic structure of Per2AS (red) in relation to Per2 (black). Dotted lines indicate the regions where the exons of Per2 and Per2AS overlap. (C) Northern blot analysis of Per2AS. PolyA ${ }^{+}$enriched RNA was extracted from mouse liver at ZT 4 and probed with Per2AS sequence (1-1812 nt that are shared in all the variants, depicted in $B)$. Numbers on the left represent the size of RNA markers. $(D)$ Subcellular localization of Per2AS and Per2 RNAs in mouse liver at ZT 4 or at ZT 16, NIH3T3 cells and MEFs. (Neat1) Nuclear RNA, (Gapdh) cytoplasmic RNA serving as controls. (E) Genome browser view on Chromosome 1 for both ribosome-bound transcripts (top) and ribosome-depleted RNAs (bottom). Data analyzed are from Janich et al. (2015). All the data represent mean $\pm \operatorname{SEM}(n=2-5)$.
(Supplemental Fig. S1A; Phatnani and Greenleaf 2006). RNAPII's recruitment pattern also coincides with the expression pattern of Per2AS (Supplemental Fig. S1B), indicating that Per2AS is transcribed by RNAPII.

Per2AS consists of at least three variants ranging in size from 2000 to $3500 \mathrm{nt}$ (Fig. 1B), while Northern blot analysis yielded a strong signal around $\sim 5000 \mathrm{nt}$ (Fig. 1C). Given that the signal from Northern blot was smeared, ranging from $\sim 1500$ to $5000 \mathrm{nt}$ (Fig. 1C), and the Per2AS signals from circadian transcriptome studies were detected in a $\geq 10-\mathrm{kb}$ range (Supplemental Fig. S1A; Koike et al. 2012; Menet et al. 2012; Zhang et al. 2014), Per2AS presumably has many variants that are different in splicing patterns and transcription termination sites.

All three Per2AS variants that we detected by RACE have a potential to encode a small protein (the longest ORF common to all three is $291 \mathrm{nt} ; 97$ amino acids); however, the potential polypeptide has no sequence similarity to any existing or predicted polypeptides in the GenBank protein database or the Conserved Domain Database. Furthermore, the Coding Potential Calculator (http://cpc.cbi.pku.edu.cn) predicts that Per2AS does not encode a protein, as the coding score of Per2AS is much smaller (from -0.97 to -0.99 depending on variant) than that of Per2 (17.53). Furthermore, Per2AS transcripts are predominantly detected in the nucleus in mouse liver both at ZT4 and ZT16 the peak and trough times of Per2AS, respectively), NIH3T3 cells, and MEFs (Fig. 1D), similar to Neat1, a lncRNA known to localize in the nucleus (Clemson et al. 2009). In contrast, proteincoding transcripts, such as Gapdh and Per2, were localized largely in the cytoplasm in order for them to be translated (Fig. 1D). In addition, Per2AS is not bound to polysomes and therefore not actively translated, in contrast to Per2 that shows strong signals both in ribosome profiling and transcriptome data, and those signals are higher at ZT16 compared with ZT4 (Fig. 1E; Supplemental Fig. S1C; Atger et al. 2015; Janich et al. 2015). The neighboring protein-coding transcript, Traf3ip1, that is transcribed from the same strand as Per2AS, also showed a clear signal both in ribosome profiling and transcriptome data, eliminating the possibility that lack of Per2AS signals in the polysome-bound fraction is due 
to its strand. These data collectively indicate that Per2AS is a long noncoding antisense transcript and does not produce a protein.

\section{Per2AS regulates Per2 and the amplitude of the circadian} clock

To understand the functional relevance of Per2AS in the mammalian circadian clock, we first used CRISPR technology to perturb Per2AS expression. We targeted the putative Per2AS promoter region, defined by the strong and rhythmic recruitment of RNAPII-Ser5P spanning 900 bp (Supplemental Fig. S1A,B). We introduced CRISPR mutagenesis in two independent cell lines: MEFs from PER2:: LUCIFERASE (PER2::LUC) knock-in reporter mice, in which a luciferase gene is fused to the $3^{\prime}$ end of the endogenous Per2 gene (Yoo et al. 2004), and Bmal1-luc, an NIH3T3-derived luciferase-reporter cell line that has been stably transfected by a luciferase gene driven by the Bmal1 promoter (Morf et al. 2012). Following singlecell sorting, we successfully isolated two PER2::LUC MEF (5D8 and 6F8) and one Bmal1-luc (mut8) mutant clones that had similar but distinct mutations at $\sim 260$ bp upstream of Per2AS TSS (Supplemental Fig. S1D). Because these cell lines were near tetraploid on average (Supplemental Fig. S1E), we not only identified the types of mutations but also calculated the frequency of each mutant allele in each mutant (Supplemental Fig. S1D). All the alleles were mutated in PER2::LUC 5D8 and $6 \mathrm{~F} 8$, in which various deletions were detected (i.e., 11 bp, 3 bp, 2 bp, and 1 bp). Meanwhile in Bmal1-luc (mut8), only $29 \%$ of alleles had a mutation (6-bp deletion) and the remaining $71 \%$ were intact (Supplemental Fig. S1D). We also targeted five other regions within the Per2AS promoter, including TSS and the TATA-box-like sequence (TATAATCAA) located $63 \mathrm{bp}$ upstream of the TSS; however, we were unable to isolate mutant clones, probably because these targeted regions were essential for cell survival or could not be easily accessed by the CRISPR machinery.

In these mutant cell lines, the level of Per2AS was upregulated to $138 \%$ (5D8) and 325\% (6F8) compared with the control (parental) cell line, as opposed to our expectations that Per2AS would be down-regulated. Nonetheless, the level of Per2 was reduced to $57 \%$ (5D8) and 70\% (6F8), respectively (Fig. 2A). Similarly, in Bmal1-luc mut8, the Per2AS level was up-regulated to $145 \%$, while the Per2 level was reduced to $76 \%$ (Fig. 2D). These data indicate that the DNA region $\sim 260$ bp upstream of the Per2AS TSS is important for Per2AS expression and that Per2AS represses Per2. Some long noncoding antisense transcripts modulate the expression of not only their target sense gene but also neighboring genes (Halley et al. 2014; Villegas et al. 2014). However, this was not the case for Per2AS, as the levels of Ilkap, Hes6, and Traf3ip1, the three closest genes, remained unchanged in all of our mutants (Supplemental Fig. S1F,G).

When we monitored the bioluminescent output from these mutant cells, the luminescent levels of both 5D8 and 6F8 were lower and their rhythmicity was less robust compared with the control (Fig. 2B; Supplemental Fig. S2A,B). Quantification analyses further revealed that the amplitude of 5D8 and 6F8 was decreased to $25 \%-30 \%$ and the period was $\sim 1.5 \mathrm{~h}$ shorter compared with the control (Fig. 2C). In contrast, the bioluminescence signal from Bmal1-luc mut8 was markedly higher (Fig. 2E; Supplemental Fig. S2C,D); the amplitude was increased to $184 \%$ and the period was $0.9 \mathrm{~h}$ shorter compared with the control (Fig. 2F).

We also measured the level of BMAL1 and PER2 proteins in these Per2AS mutants and found that the level of BMAL1 protein was slightly elevated in all three mutants (Fig. 2G,H), consistent with the results from Bmal1-luc reporter output (Fig. 2E). We were unable to detect PER2::LUC protein (data not shown); however, PER2::LUC is a fusion protein in which a luciferase gene is fused in-frame to the $3^{\prime}$ end of the endogenous mouse Per2 gene, and bioluminescent output is considered to represent the amount of PER2::LUC protein (Yoo et al. 2004; Chen et al. 2009; Yoo et al. 2017). In contrast, PER2 protein level was moderately increased in Bmal1-luc mut8 cells (Fig. 2G,H), despite the fact that the Per2 mRNA level was decreased in this cell line. There are two plausible reasons to explain the increase in PER2: an off-target effect of CRISPR, or the compensation mechanism as the level of PER2 is critical to maintain circadian rhythmicity (Chen et al. 2009). We think the former unlikely, as none of the potential hits for off-targets in exon included clock genes, and translational regulation of PER2 has not been reported thus far, although we cannot fully exclude this possibility. We also think that changes in period are most likely due to the effect of Per2AS on Per2, as the period of a luciferase reporter output is shorter in mouse fibroblasts (Ramanathan et al. 2014), although the effect of Per2 on circadian period in Per2 $\mathrm{KD} / \mathrm{KO}$ is highly variable between species, tissues, its expression level, and type of measurements (see the Discussion for more details).

To further characterize the Per2AS mutants as well as to gain insights into the underlying mechanisms of circadian amplitude regulation, we next measured the mRNA expression patterns of 13 core clock genes after synchronizing these cells by serum shock (Balsalobre et al. 1998). We found that the mRNA expression of Bmal1, Cry1, and Rora were elevated, while the expression of Per2 was decreased in all three mutants (Fig. 3). The upregulation of Bmal1 mRNA level was consistent with the increased bioluminescence levels in Bmal1-luc cells and BMAL1 protein level (Fig. 2E,G,H). The mRNA expression of Nfil3 was up-regulated in PER2::LUC 5D8 and Bmal1-luc mut8, while Cry2 levels are higher in 5D8 and 6F8 than those in WT (Fig. 3, bottom left). Interestingly, the expression of Npas2 was down-regulated in the PER2::LUC mutants but up-regulated in the Bmal1luc mutant. No significant changes were observed for Rev-erb $\alpha$ and Rev-erb $\beta$ (Fig. 3). These data suggest that Bmal1, Cry1, Rora, Per2 (changed in all three mutants), but not $R e v$-erb $\alpha / \beta$, are involved in the Per2AS-mediated amplitude regulation. 
A
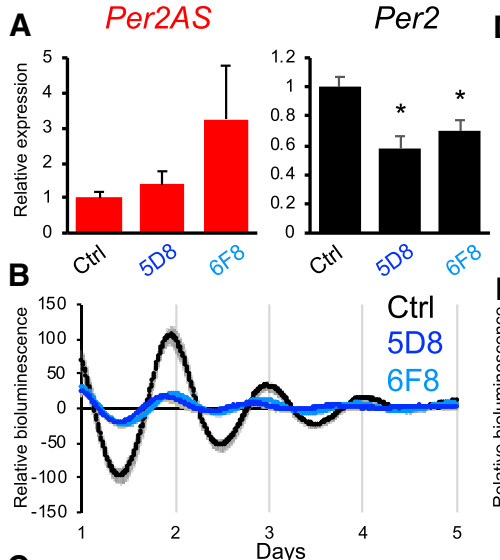

C

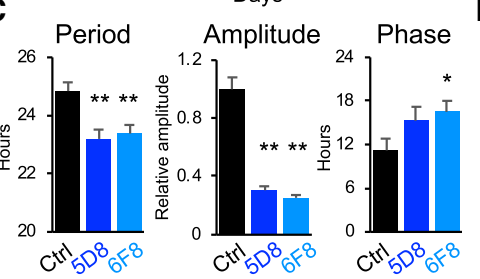

D Per2AS
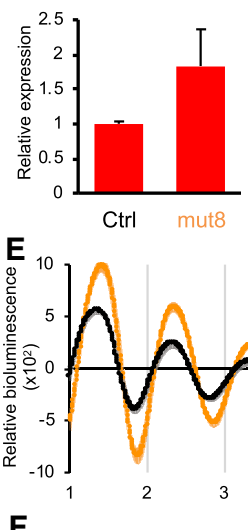

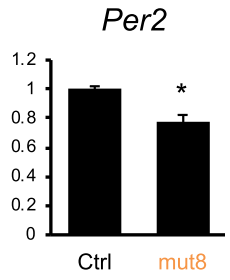

Period Amplitude Phase

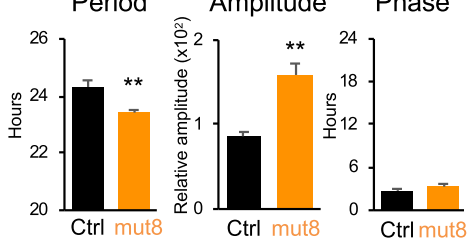

Figure 2. Per2AS mutants alter Per2 and the amplitude of the circadian clock. $(A, D)$ Relative expression levels of Per2AS (red), and Per2 (black) in the Per2AS mutant cell lines of PER2::LUC MEFs $(n=4-6)(A)$ or Bmal1-luc $(D)$ $(n=13)$. $(B, E)$ Detrended bioluminescent output of the mutant cell lines of PER2::LUC MEFs (WT: $n=13$, 5D8: $n=13$, 6F8: $n=13$ ) (B) or Bmal1-luc (WT: $n=23$, mut8: $n=23)(E)$. Bold lines represent the mean, while shaded areas represent the SEM. $(C, F)$ Period (left), amplitude (middle), and phase (right) of bioluminescent output calculated from $B$ (PER2::LUC MEFs) or $E$ (Bmal1-luc). (G) Representative images of Western blot analyses. $(H)$ Quantification of BMAL1 and PER2 levels from Western blot analyses. All the data represent mean \pm SEM. $\left.\left(^{*}\right) P<0.05,{ }^{* *}\right) P<0.005$ (Student's $t$-test).
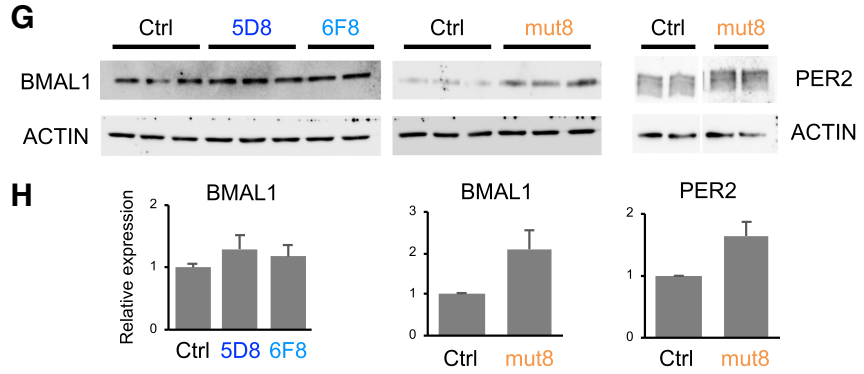

Transcripts of Per2AS do not play a major role in the circadian clock machinery

In contrast to coding genes whose functional unit is an encoded protein, the functional unit of lncRNAs is often unknown. It can be the transcript itself (i.e., RNA molecule and/or the small ORF embedded in the sequence that has the potential to encode a protein) that regulates target mRNAs post-transcriptionally by affecting splicing, mRNA stability, mRNA localization, or epigenetic marks (Wight and Werner 2013; Khorkova et al. 2014). It can also be the very act of transcription, in which transcription of one strand suppresses transcription of another in cis, a process called transcription interference (Wight and Werner 2013; Khorkova et al. 2014).

To distinguish whether the functional unit of Per2AS is either a transcript (i.e., post-transcriptional model) or the act of transcription (i.e., pretranscriptional model) (Battogtokh et al. 2018), we post-transcriptionally decreased the level of Per2AS using "gapmers," chimeric antisense nucleotides containing modified nucleic acid residues to induce RNase H-mediated degradation of nuclear-retained RNA (Lee et al. 2012), as the majority of Per2AS RNA remains in the nucleus in fibroblasts (Fig. 1D) and RISC-mediated RNA cleavage triggered by siRNAs occurs mainly in the cytoplasm (Carthew and Sontheimer 2009). We

also targeted gapmers to exon 1 of Per2AS (i.e., intron 6 of Per2) that was shared by all three variants (Fig. 1B). Gapmers 5 and 8 successfully reduced the level of Per2AS to $61 \%$ in Bmal1-luc cells (Fig. 4A); however, the level of Per2 remained unchanged (Fig. 4A). Even though gapmers can not only induce RNA duplex-mediated degradation but also premature transcriptional termination of target mRNAs leading to reduced transcriptional activity and thereby confounding the interpretation of the results (Lee and Mendell 2020), this is unlikely the case for Per2AS gapmers, as the level of Per2 is unaffected. Unexpectedly, however, gapmer-mediated Per2AS knockdown led to an $\sim 20 \%$ reduction in the Bmal1 level in Bmal1-luc cells (Fig. 4A).

We also post-transcriptionally overexpressed Per2AS using variant 2 (i.e., the longest variant) (Fig. 1B) in Bmal1-luc cells. This led to a marked increase in the Per2AS level by 75,000-fold. Nevertheless, the Per2 level remained unchanged, whereas the Bmal1 level showed an approximate twofold increase (Fig. 4B). Knockdown or overexpression of Per2AS did not alter period, phase, or amplitude of bioluminescence output in Bmal1-luc cells (Fig. 4C). The change in Bmal1 level was not due to comparable changes in the levels of Rev-erb $\alpha / \beta$ or Ror $\alpha$, transcription repressor and activators of Bmal1, respectively (Fig. 4A,B; Sato et al. 2004). Rather, Per2AS appears 
Mosig et al.

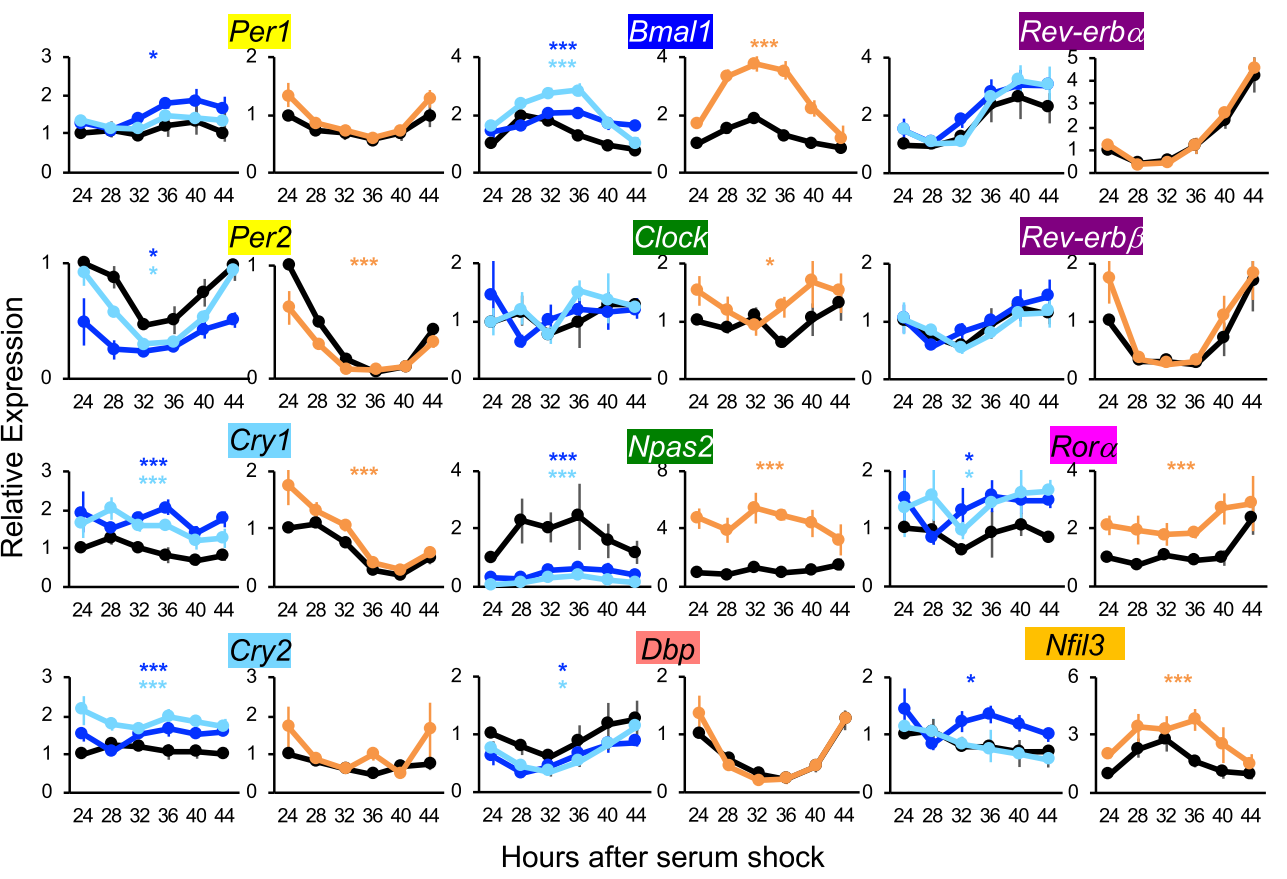

Figure 3. Relative expression levels of core clock genes in the Per2AS mutant cell lines. All the data represent mean SEM. PER2::LUC MEFs (WT: $n=3,5$ D8: $n=3,6$ F8: $n=3$, color key in Fig. 2B) or Bmal1-luc (WT: $n=3$, mut8: $n=3$, color key in Fig. $2 \mathrm{E}) .\left({ }^{*}\right) P<0.05,\left({ }^{* * *}\right) P<$ 0.005 (two-way ANOVA).

to directly regulate the Bmal1 transcription, as the increasing amount of Per2AS RNAs led to up-regulation of reporter activity of Bmal1-luc but not Per2-luc (Fig. 4D). Interestingly, however, the changes of Bmal1 by Per2AS RNA was also cell type-specific, as it was not observed in PER2::LUC MEFs, even though the efficiency of Per2AS knockdown was higher (gapmer 5: 63\%, gapmer 8: 60\%) in PER2::LUC MEFs, compared with Bmal1-luc cells (both gapmers 5 and 8: 39\%) (Supplemental Fig. S3). Overall, these data indicate that the transcript of Per2AS has little effect on the circadian control system (i.e., that its effects are not post-transcriptional). However, we also observed a small effect of Per2AS on Bmal1 at least in Bmal1-luc cells, suggesting that Per2AS post-transcriptionally regulates Bmal1 in a cell-specific manner.

\section{Per2 knockdown down-regulates Per2AS and does not} replicate the phenotypes of Per2AS CRISPR mutants

If Per2AS and Per2 indeed form a double negative feedback loop and mutually inhibit each other's expression, then Per2 mRNA knockdown would result in up-regulation of Per2AS level in the post-transcriptional model but no changes in the pretranscriptional model. To test this, we first reduced the level of Per2 post-transcriptionally using shRNAs. Our Per2 knockdown successfully reduced the level of Per2 to $65 \%$ in AML12 cells, a mouse hepatocyte cell line, and $45 \%$ in Bmal1-luc cells (Fig. 5A,F). Per2 knockdown also led to a decrease in the amplitude of bioluminescence output in PER2::LUC MEFs but not in Bmal1-luc cells (Fig. 5B-D). Period remained unchanged upon Per2 knockdown in both cells, despite that previous studies in murine fibroblast and hepatocyte cell lines reported that circadian period became shorter upon Per2 knockdown (Ramanathan et al. 2014). The residual Per2 level is higher in our system compared with those reports $(45 \%$ vs. $20 \%)$, and this could have contributed to the difference observed in our study. Nevertheless, Per2 knockdown resulted in a decrease of the Per2AS level to $55 \%$ (Fig. 5A), contrary to either of our expectations (i.e., pretranscriptional or post-transcriptional interference).

We also measured the expression patterns of the core clock genes upon Per2 knockdown in Bmal1-luc cells to evaluate whether the changes observed in the Per2AS mutant cells (Fig. 3) were mediated entirely through Per2AS's effect on Per2. Per2 knockdown did not result in changes of Bmal1 mRNA levels (Fig. 5F), consistent with the bioluminescent output from Bmal1-luc cells (Fig. 5D) but inconsistent with our observations in Bmal1-luc mut8 cells, in which Bmal1-luc bioluminescence and Bmal1 mRNA level were both significantly increased (Figs. 2E, 3). In addition, changes in mRNA expression patterns were observed only for Nr1d2 and Nfil3 upon Per2 knockdown (Fig. 5F), in contrast to the changes in the mRNA expression patterns of Cry1, Bmal1, Clock, Npas2, Rora, and Nfil3 observed in Bmal1-luc mut8 cells (Fig. 3). Interestingly, we did not observe any changes in mRNA levels of the core clock genes upon Per2 knockdown in AML12 cells (Supplemental Fig. S4A), in which the level of Per2AS is $\sim 100$-fold higher compared with Bmal1-luc cells (Supplemental Fig. S4B). As the level of Per2 was lower in Bmal1-luc with Per2 knockdown (45\%) compared 
A Per2AS knock-down

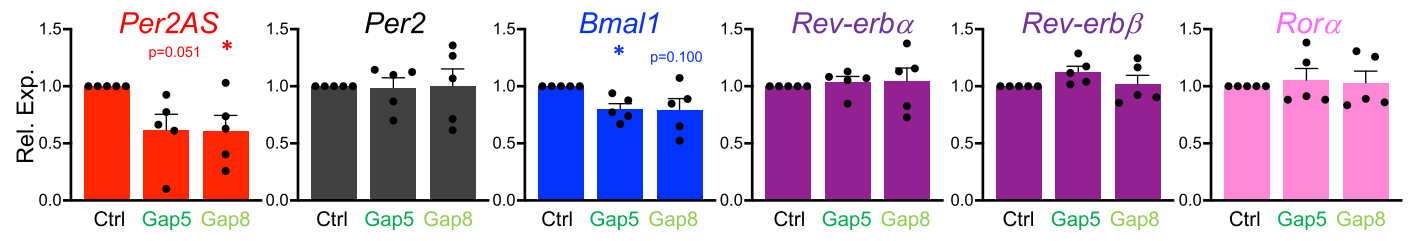

\section{B Per2AS overexpression}
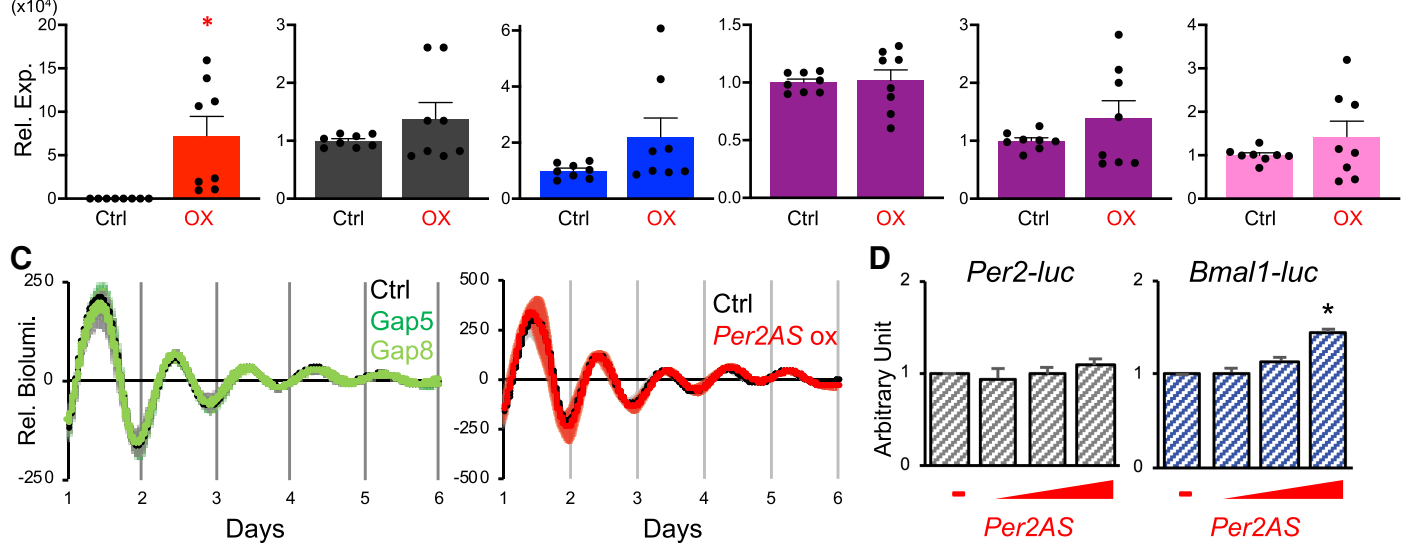

Figure 4. Per2AS transcripts regulate Bmal1 but not Per2 in Bmal1-luc cells. (A) Relative expression levels of core clock genes normalized by $36 B 4$ upon Per2AS knockdown $(n=5)$. The values for controls were used as a reference and set at 1 . $(B)$ Relative expression levels of core clock genes normalized by $36 B 4$ upon Per2AS overexpression $(n=7-8)$. All the data represent mean \pm SEM. $\left({ }^{*}\right) P<0.05$. We excluded the samples that had $<5000$-fold induction of Per2AS. (C) Bioluminescent output from Bmal1-luc cells upon knockdown $(n=8)$ or overexpression $(n=4)$ of Per2AS. Bold lines represent the mean, while shaded areas represent the SEM. $(D)$ Luciferase activity from Per2-luc or Bmal1-luc cotransfected with an increasing amount of Per2AS (variant2)-expressing plasmid.

with Bmal1-luc mut8 cells $(75 \%)$, it is highly unlikely that changes in the mRNA expression patterns observed in Bmal1-luc mut8 cells (Fig. 3) are solely due to the Per2$A S^{\prime}$ s effect on Per2. Rather, these data support the idea that Per2AS has a distinct role in the mammalian circadian system, independent of Per2.

Overall, our study has highlighted several important functions and regulatory mechanisms of Per2AS in the mammalian circadian clock system (Fig. 6): (1) Per2AS regulates circadian amplitude, (2) Per2AS employs both transcript- and transcription-dependent mechanisms, (3) the effect of Per2AS is both Per2-dependent and-independent, and (4) some of the functions and regulatory mechanisms of Per2AS are cell type-specific. Although the amplitude is an integral part of any cycling system, the molecular mechanisms of circadian amplitude regulation have remained enigmatic. Deepening our understanding how Per2AS functions in the circadian system will help us gain important insights into how the amplitude is regulated in the future.

\section{Discussion}

Based on the inverse expression patterns of Per2AS and Per2, we originally hypothesized that Per2AS and Per2 mutually inhibit each other's expression and form a double negative feedback loop for Per2AS to exert its function. The experimental observations from this study bear out the assumptions underlying our mathematical models of Per2-Per2AS interactions, in which Per2AS and Per2 mutually inhibit each other either transcriptionally or posttranscriptionally, or a combination of both effects (Battogtokh et al. 2018). Our experimental interrogation clearly demonstrated that Per2AS represses Per2 (Fig. 2A,D), presumably via a transcriptional mechanism, as the knockdown or overexpression of Per2AS transcripts did not alter the level of Per2 mRNAs (Figs. 4, 6). Contrary to our expectation, however, Per2 positively regulates Per2AS, as the knockdown of Per2 led to down-regulation (rather than up-regulation) of Per2AS (Fig. 5A). This effect is post-transcriptional, because the level of Per2 premRNA remained unchanged upon Per2 knockdown (Supplemental Fig. S4C). We think it is unlikely that Per2 RNA and Per2AS RNA form an RNA duplex and stabilize each other, because (1) the expression of Per2AS and Per2 are antiphasic in at least some tissues (Fig. 1A; Zhang et al. 2014), (2) Per2 is 25 times more abundant than Per2AS (Fig. 1A; Koike et al. 2012), (3) Per2 predominantly localizes in the cytoplasm while Per2AS localizes in the nucleus (Fig. 1D), and (4) Per2AS consists of many variants and their nucleotide sequences vary (Fig. 2E). Rather we favor the hypothesis that PER2 indirectly or directly regulates Per2AS transcription. In fact, REV-ERB $\alpha / \beta$ and BMAL1/ CLOCK/PER1/PER2/CRY2 are recruited to the vicinity of the Per2AS TSS (Koike et al. 2012), potentially involved in regulating Per2AS transcription. Regardless of the mechanism, Per2 is the only core clock gene whose 
A

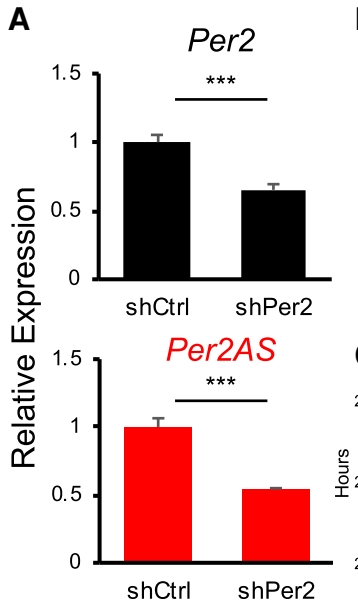

$\mathbf{F}$

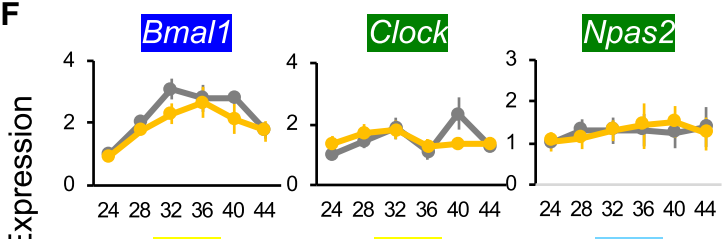

B

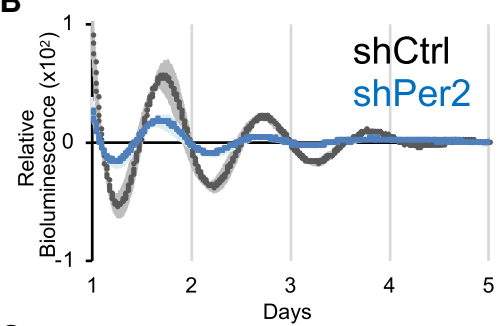

D

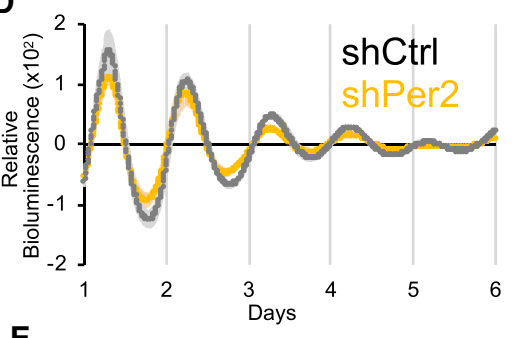

E Period Amplitude Phase
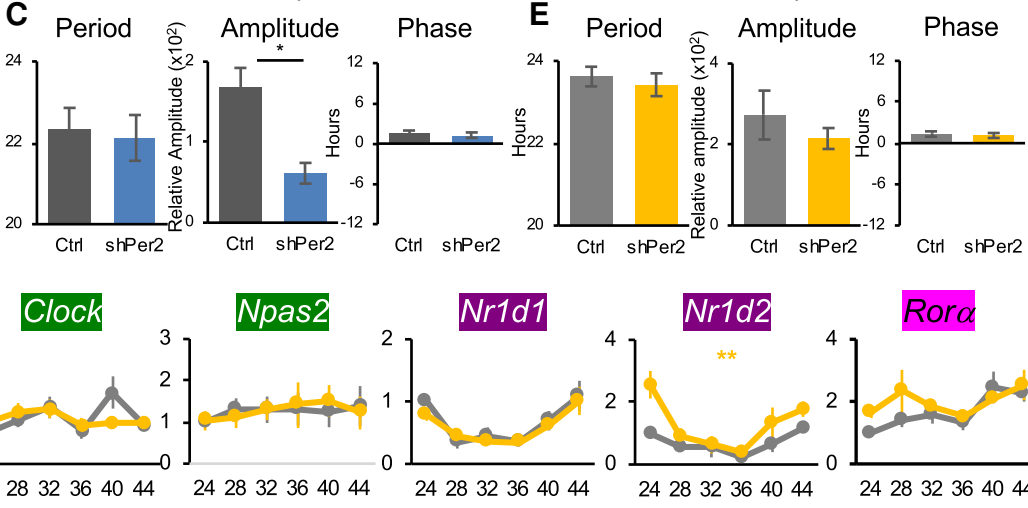

Nr1d2

Rora

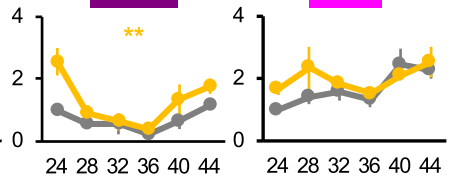

Cry1

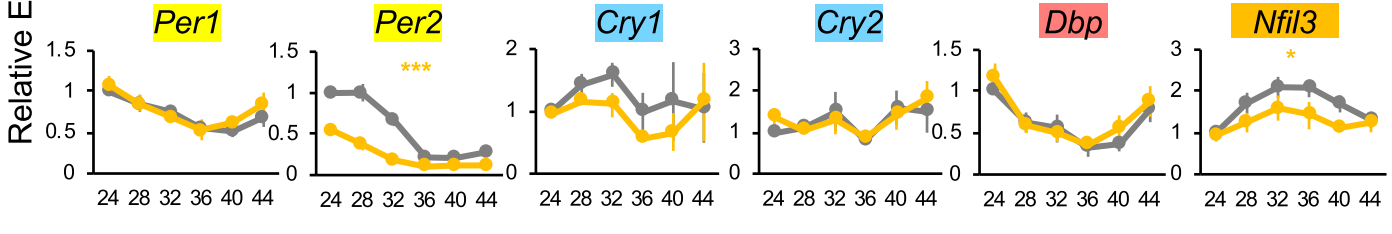

Dbp

Nfil3

Hours after serum shock

Figure 5. Per2 knockdown does not replicate the phenotypes of Per2AS mutants. (A) Relative expression levels of Per2 (top) and Per2AS (bottom) in the AML12 mouse hepatocytes $(n=4-5) .(B, D)$ Relative bioluminescence output from PER2::LUC MEFs $(B)(n=12)$ or $B m a l 1$ luc $(D)(n=14)$ upon Per2 knockdown. (C,E) Period (left), amplitude (middle), and phase (right) of bioluminescent output calculated from $B$ (PER2::LUC MEFs) or D (Bmal1-luc). ( F) Relative expression levels of core clock genes in Bmal1-luc cells upon Per2 knockdown $(n=3-5)$. All the data represent mean \pm SEM. $\left({ }^{*}\right) P<0.05,(* * *) P<0.005$ (two-way ANOVA).

rhythmicity, proper phase, and expression levels are all critical to sustain rhythmicity (Zheng et al. 1999; Bae et al. 2001; Chen et al. 2009). This study demonstrates that Per2AS serves as an additional regulatory mechanism to further ensure that Per2's expression pattern stays within a certain window for sustaining robust circadian rhythmicity.

Our mathematical models were also constrained by the requirement that the expression of Per2AS and Per2 are both rhythmic and antiphasic ( $180^{\circ}$ out of phase). These patterns have been observed not only in liver but also in adrenal gland, lung, and kidney (Zhang et al. 2014). Analysis of mouse ENCODE data sets demonstrated that Per2AS is also expressed in many other tissues, such as genital and subcutaneous fat pad, bladder, kidney, colon, duodenum, large and small intestines, stomach, lung, and potentially in mammary gland, ovary, and testis (Supplemental Fig. S5A; The ENCODE Project Consortium 2012; Davis et al. 2018), although it is unclear whether Per2AS expression is rhythmic and antiphasic to Per2 in these tissues. A Per2AS signal has not been detected in the central nervous system and a few other peripheral tissues including heart, placenta, spleen, and thymus (Sup- plemental Fig. S5A; The ENCODE Project Consortium 2012; Davis et al. 2018). These results could be due to tissue sampling times (i.e., little or no Per2AS may have been expressed when these tissues were harvested), or the Per2AS level was under the detection threshold, as the expression of lncRNAs is generally low $>>10$-fold lower than sense transcripts on average) and transcriptome analyses sometimes lack the sensitivity to detect all lncRNAs (He et al. 2008; Faghihi and Wahlestedt 2009; Xu et al. 2009; Xu et al. 2011; Diebali et al. 2012). Nevertheless, we recently reported that the level of Per2AS linearly correlates with that of Rorc, as well as the percentage of cycling genes among 12 mouse tissues (Littleton et al. 2020), indicating that Per2AS regulates the amplitude of the circadian transcriptome in these tissues, potentially with Rorc. These data also suggest that the functions of Per2AS is not limited to the cellular level (i.e., fibroblasts) but also extended to the physiological level. An antisense transcript of Bmal1 was also detected in a few tissues, such as subcutaneous fat, bladder, kidney, colon, lung, and potentially in duodenum, stomach, mammary gland, and ovary (Supplemental Fig. S5B; The ENCODE Project Consortium 2012; Davis et al. 2018), as was also reported 


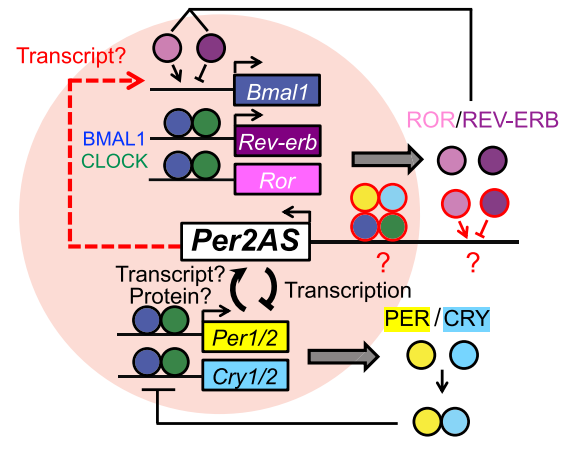

Figure 6. Putative model for the circadian molecular circuit including Per2AS. Per2AS and Per2 form a single negative feedback where Per2AS limits the expression of Per2 presumably pretranscriptionally. Per2AS can also activate the Bmal1 expression post-transcriptionally (i.e., in trans) in a tissue- or cell-specific manner. Circadian transcription factors as well as PER-CRY protein complex may be recruited to the vicinity of the Per2AS TSS (Koike et al. 2012) to potentially regulate Per2AS transcription.

previously (Zhang et al. 2014). It would be of great interest to analyze whether the antisense transcript of Bmal1 exerts some functions in the mammalian circadian clock system.

We also found that Per2AS regulates the amplitude of the circadian clock; however, Per2AS perturbation had an opposite effect on amplitude: a decrease in PER2:: $L U C$ reporter but an increase in Bmal1-luc reporter oscillations (Fig. 2). Because the amplitude is determined by the amplitude of either synthesis or degradation rates or a combination of both (Luck et al. 2014), the decrease of PER2::LUC reporter oscillations in the Per2AS mutants can be explained by the decreased amplitude of the PER2::LUC protein synthesis, triggered by the reduction of amplitude of Per2 mRNA oscillation (Fig. 3). Interestingly, this is in spite of the increased level of BMAL1 protein (Fig. 2). In fact, the level of Per2 pre-mRNA (an indicator of Per2 mRNA synthesis) was reduced in all the mutant cell lines (Supplemental Fig. S7), evidence that Per2 mRNA synthesis was decreased in Per2AS mutant cells. On the other hand, the increase of the amplitude of Bmal1-luc reporter oscillations is presumably due to the increase of Bmal1 mRNA synthesis rate (Fig. 3).

The molecular basis of how Per2AS regulates amplitude still remains elusive. Recent studies have revealed that lncRNAs regulate target gene expression both via their transcripts and the act of transcription: Transcripts of lncRNA can regulate target gene expression by interacting with chromatin complexes, transcription factors, or their cofactors, whereas the act of transcription can also alter local chromatin structure as transcription machinery moves along DNA during transcription elongation or facilitate chromatin loop formation between transcription machinery at enhancers and promoters of distantly located target genes (Chen 2016; Marchese et al. 2017; Kaikkonen and Adelman 2018). Indeed, the act of transcription of Per2AS is necessary to regulate Per2, while both the transcript and the act of transcription are important to regu- late Bmal1. Furthermore, the effect of the Per2AS transcript on Bmal1 is cell type-specific (Fig. 4A,B; Supplemental Fig S3A), whereas that of transcription is presumably mediated by Rora, a Bmal1 activator (Figs. 3, 6). Interestingly, there seem to be consistent effects on RORE-containing genes in the Per2AS mutants (Fig. 3), and this effect appears to be independent of Per2. Per2 knockdown leads to decreased levels of RORE-controlled genes, such as Bmal1, Cry1, and Nfil3 (Shearman et al. 2000; Schmutz et al. 2010; Ramanathan et al. 2014), whereas the levels of RORE-containing genes were up-regulated in the Per2AS mutants (Fig. 3), even though both conditions led to the decreased Per2 level. In addition, the BMAL1 protein level was increased in the Per2AS mutants (Fig. 2) but remains unchanged with Per2 KD (Supplemental Fig. S4D,E).

Our potential candidate for understanding the mechanisms of Per2AS-mediated amplitude regulation is Rors. Recent studies have also highlighted the role of the positive loop (Clock-Arntl-Rev-Ror) as the central axis of amplitude regulation (He et al. 2016; Zhao et al. 2016), and Ror genes have been shown to increase the circadian amplitude of molecular and behavioral rhythms (He et al. 2016). Furthermore, our recent study suggested that Per2AS and Rorc function synergistically in the circadian clock system and regulate the amplitude of circadian transcriptome output (Littleton et al. 2020). Understanding how transcript or transcription of Per2AS regulate RORE-containing genes may be the key to decipher how circadian amplitude is regulated.

We also observed phenotypic changes in period in addition to amplitude in the Per2AS mutants (Fig. 2), although it is unclear whether this is due to the changes in the level of Per2 or a Per2AS-specific effect independent of Per2. The period phenotype observed by Per2 $\mathrm{KD} / \mathrm{KO}$ is highly variable between species, tissues, its expression level, and type of measurements (Ramanathan et al. 2014). For example, the period of a luciferase reporter output is shorter in NIH3T3 (mouse fibroblast) and MMH-D3 (mouse hepatocyte) cells, while it remains unchanged in 3T3-L1 (mouse adipocyte) cells when the Per2 mRNA level was reduced to $20 \%-40 \%$ compared with the control (Ramanathan et al. 2014). However, the period is longer but with low amplitude in U2OS cells (human osteosarcoma cell line) when the PER2 mRNA level is reduced to $20 \%-40 \%$ compared with the control (Baggs et al. 2009; Zhang et al. 2009). In Per2 ${ }^{-/-}$mouse fibroblasts, the reporter output is arrhythmic and thus the period cannot be reliably quantified (Liu et al. 2007). In Per2 ${ }^{-/-}$tissues, the period of Per1-luc bioluminescent output is shorter in the SCN, whereas it remained unchanged in pituitary or lung (Pendergast et al. 2010). The short period phenotype is not observed in the SCN of Per2 ${ }^{+/-}$animals (Pendergast et al. 2010). The free-running period of locomotor activity is either shorter or arrhythmic in Per2 $^{-/-}$mice (Zheng et al. 1999; Bae et al. 2001; Pendergast et al. 2010; Tamiya et al. 2016), while it is unchanged in $\mathrm{Per2}^{+-}$mice (Pendergast et al. 2010; Tamiya et al. 2016). It is also worth noting that the Per2AS locus is disturbed in these $\mathrm{Per}^{-/-}$and Per2 ${ }^{+-}$animals, confounding 
the interpretation of the data (Zheng et al. 1999; Bae et al. 2001). These data collectively demonstrate that the relationship between Per2 and the circadian period is not always as clear and straightforward as has been perceived and needs to be investigated further, with consideration of the interaction between Per2AS and Per2.

These experimental observations clearly indicate that our mathematical models need to be revised to better understand the functions and regulatory mechanisms of Per2AS. Specifically, the relationship between Per2AS and Per2 is a single negative feedback loop, rather than a double negative feedback loop as we originally hypothesized. We also need to include the effect of Per2AS on $B$ mal1, as well as the potential effect of circadian transcription factors on Per2AS transcription (Fig. 6). Interestingly, a simpler mathematical model that involves only the core negative feedback loop where Per2AS and Per2 mutually inhibit each other (Supplemental Fig. S6) supports the predictions from our original model, in which Per2AS would make the circadian oscillations more robust (Battogtokh et al. 2018). It will be of future interest to test whether the revised model also leads to the same conclusion.

Antisense transcripts of a core clock gene have also been reported in other organisms. In Neurospora, the sense frequency $(f r q)$ and antisense $(q r f)$ transcripts are both located on the same chromosome and overlap almost completely (Kramer et al. 2003; Xue et al. 2014). In Anther$a e a$, the sense-antisense transcripts for the homolog of Drosophila's period (per) gene are located on different chromosomes (Sauman and Reppert 1996). Interestingly, human PER2 also has an antisense transcript (PER2AS), but, based on human ENCODE data sets, the transcription of PER2AS and PER2 diverges from their respective TSSs, most likely using a bidirectional promoter (Supplemental Fig. S5C; The ENCODE Project Consortium 2012; Davis et al. 2018). Although it is unclear whether all these sense-antisense transcripts have rhythmic and antiphasic expression patterns, the evolutionary conversation of sense-antisense pairs of a core clock gene suggests that antisense transcripts are part of a common mechanism for circadian clock regulation.

Even though the expression of $q r f$ is rhythmic and antiphasic to $f r q$, similar to Per2AS and Per2 (Kramer et al. 2003; Xue et al. 2014), their functions in the core clock machinery appear to be different. Frq and qrf form a double negative feedback loop, and the primary function of $q r f$ is to regulate phase and light entrainment in the presence of light and amplitude in the absence of light (Kramer et al. 2003; Xue et al. 2014). Because the molecular clock circuitry is markedly more complex in mammals than Neurospora, it is possible that Per2AS acquired additional or different functions, such as its apparent ability to regulate Bmal1 in trans.

There is only limited experimental evidence about the functions and regulatory mechanisms of antisense transcripts, particularly in mammals. Most of the effort has been concentrated on understanding the function of antisense transcripts as an RNA molecule (transcript), and only a few studies have tested their function via transcriptional interference to regulate the sense gene expression.
In addition, most of the studies have not analyzed the potential impact of the sense to the antisense transcript/transcription, despite the reciprocal regulation between sense and antisense transcripts being the most biologically intuitive mechanism. Our study contributes to an understanding of the possible functional roles of antisense transcripts by demonstrating that Per2AS is an important molecule to not only maintain the level of Per2 within the oscillatory range but also to regulate the amplitude of the circadian clock. It would be of great interest to explore the functions of Per2AS at the tissue and organismal levels in the future.

\section{Materials and methods \\ Tissue harvesting and cell culture}

Male C57BL/6J mice were maintained on a 12:12 LD cycle and fed ad libitum, and livers were collected at the time indicated. All the animal experiments were conducted according to the protocols approved by the Institutional Animal Care and Use Committees at the University of Texas Southwestern Medical Center.

NIH3T3, HEK293/T17, mouse embryonic fibroblasts (MEFs), PER2::LUC MEFs, and Bmal1-luc cells were grown in Dulbecco's modified Eagle medium (Life Technologies) with $10 \%$ fetal bovine serum (FBS) (Atlanta Biologicals) at $37^{\circ} \mathrm{C}$ with $5 \% \mathrm{CO}_{2}$. AML12 cells were grown in Dulbecco's modified Eagle medium/F12 (1:1) with $10 \%$ FBS and $1 \%$ insulin-transferrin-selenium supplement (Gibco) at $37^{\circ} \mathrm{C}$ with $5 \% \mathrm{CO}_{2}$.

Chromosomal numbers were counted as previous reported (Nicholson et al. 2015) with minor modifications. Briefly, cell cultures were incubated in $1000 \mathrm{ng} / \mathrm{mL}$ Colcemid (Karyomax, Invitrogen) for $3-6 \mathrm{~h}$ at $37^{\circ} \mathrm{C}$ to enrich in mitotically arrested cells. The cells were then collected by centrifugation, and prewarmed hypotonic solution $(0.075 \mathrm{M} \mathrm{KCl})$ was added drop-wise to the cell pellet. After incubation for $18 \mathrm{~min}$ at $37^{\circ} \mathrm{C}$, cells were fixed with an ice-cold 3:1 methanol:acetic acid solution for $5 \mathrm{~min}$ followed by centrifugation. After repeating this last step twice, fixed cells were dropped on microscope slides.

\section{CRISPR mutagenesis}

The sgRNAs were designed by the CRISPR design tool (http ://crispr.mit.edu), and complementary oligonucleotides were annealed, phosphorylated, and cloned into the BbsI sites of the pSpCas9(BB)-2A-GFP (Addgene plasmid 48138) (Ran et al. 2013). After the nucleotide sequences of the plasmids were verified by Sanger sequencing, DNA was transfected into PER2::LUC MEFs and/or Bmal1-luc cells using FuGENE6 according to the manufacturer's instructions. After $48 \mathrm{~h}$, cells were trypsinized and sorted using FACSAria I (BD Biosciences) with GFP signals. Subsequently, cells were cultured in DMEM supplemented with $10 \%$ FBS and $1 \times$ penicillin/streptomycin (Gibco). When each clone reached confluency, cells were lysed with $90 \mu \mathrm{L}$ of $50 \mathrm{mM} \mathrm{NaOH}$ and $10 \mu \mathrm{L}$ of $100 \mathrm{mM}$ Tris- $\mathrm{HCl}(\mathrm{pH} 8.0$ ), followed by incubation for $10 \mathrm{~min}$ at $95^{\circ} \mathrm{C}$. The mutated genomic region was first amplified by PCR, then cloned into the pGEM-T vector (Promega), to reveal the nucleotide sequence of each clone by Sanger sequence. All the primer sequences used in this study are in Supplemental Table S1.

\section{Real-time bioluminescence recordings and luciferase assay}

Cells were first plated into $35-\mathrm{mm}$ dishes and then allowed to become confluent. The medium was then replaced with DMEM 
supplemented with $50 \%$ horse serum (gene expression analyses) or dexamethasone $(1 \mu \mathrm{M})$ was added to the media (real-time bioluminescence recordings) for $2 \mathrm{~h}$. After synchronizing cells, medium was changed to phenol red-free DMEM (Cellgro 90-013-PB) supplemented with $100 \mu \mathrm{M}$ luciferrin, $10 \mathrm{mM}$ HEPES ( $\mathrm{pH} 7.2$ ), $1 \mathrm{mM}$ sodium pyruvate, $0.035 \%$ sodium bicarbonate, $2 \%$ FBS, $1 \times$ penicillin/streptomycin, and $2 \mathrm{mM}$ L-glutamine. Real-time bioluminescence recordings were performed using a LumiCycle (Actimetrics, Inc.). Quantification analyses was performed by JMP software (Oklejewicz et al. 2008). The recordings from the first $24 \mathrm{~h}$ were eliminated for quantitative analyses because these data are unreliable.

Luciferase assays were performed as previously reported (Kojima et al. 2010). Briefly, a mixture of plasmid DNAs containing $100 \mathrm{ng}$ of Per2E2-luc or Bmal1-luc firefly luciferase reporter genes, $10 \mathrm{ng}$ of Renilla luciferase reporter genes, and increasing amounts of Per2AS (variant2)-expressing plasmids (50, 100, 200, and $400 \mathrm{ng}$ ) were cotransfected in NIH3T3 cells. Luciferase activities were measured $\sim 48 \mathrm{~h}$ after transfection. The Per2E2-luc reporter gene was constructed by inserting the mouse Per2 promoter $(-83$ to +156 in reference to the Per2 TSS) into pGL4.12[luc2CP] (Promega), whereas Bmal1-luc was constructed by inserting the mouse Bmal1 promoter $(-779$ to +127 in reference to the Bmal1 TSS) into pGL4.11[luc2P] (Promega).

RT-qPCRTotal RNA was extracted with TRIZOL reagent (Life Technologies) according to the manufacturer's instructions and treated with TURBO DNaseI (Life Technologies). RNAs were then subjected to reverse transcription using SuperScript II (Life Technologies) or high-capacity cDNA reverse transcription kits (Applied Biosystems). For Per2AS transcripts, cDNA was synthesized using strand-specific primers (Supplemental Table S1), except for Figure 1A, in which the oligo(dT) was used. qPCR was performed using QuantiStudio 6 (Life Technologies) with SYBR Power Green (Applied Biosystems). All the primer sequences used in this study are in Supplemental Table S1.

\section{Rapid amplification of cDNA ends assay}

A RACE assay was performed with a SMARTer RACE $5^{\prime} / 3^{\prime}$ kit (Clontech) according to the manufacturer's instructions. Total RNAs from mouse liver (C57BL/6J) harvested at ZT4 was subjected to $5^{\prime}$ - and 3'-RACE cDNA synthesis. Each cDNA was then amplified by Per2AS-specific primers (Supplemental Table S1) together with the Universal Primer (Clontech). The first PCR products were subsequently amplified by the nested Per2AS-specific primers with Universal Primer (Clontech). The nested PCR products were cloned into the pGEM-T vector (Promega), and the nucleotide sequence was determined by Sanger sequencing. Two independent $5^{\prime}$ RACE products had the identical sequence, while four $3^{\prime} \mathrm{RACE}$ products yielded three different sequences, giving rise to the three variants of Per2AS (Fig. 1B; Supplemental Fig. S1C).

\section{Northern blotting}

Total RNA ( 500 $\mu \mathrm{g})$ extracted from mouse liver harvested at ZT4, at which Per2AS expression is the highest (Koike et al. 2012), was subjected to a poly(A) ${ }^{+}$tract isolation kit (Promega). Poly $(\mathrm{A})^{+}$enriched RNA, as well as the RNA ladder (Life Technologies), were further separated on a $1 \%$ agarose gel, transferred to Hybond- $\mathrm{N}^{+}$membrane (Amersham), and UV cross-linked (Stratagene) using a standard protocol. The membrane was further hybridized overnight at $68^{\circ} \mathrm{C}$ using PerfectHyb PLUS (Sigma) with a $\mathrm{P}^{32} \mathrm{UTP}$-labeled probe that has a complementary sequence against Per2AS (1-1812 nt) (Fig. 1B; Supplemental Fig. S1C), where all the variants share its nucleotide sequences. After extensive washing of the membrane, the radioactive signal was analyzed with a Storm image analyzer (GE Healthcare).

\section{Subcellular fractionation}

Livers from mice (C57BL/6J) were immediately rinsed with an ice-cold $0.9 \% \mathrm{NaCl}$ solution, then homogenized on ice with five volumes of homogenization buffer $(10 \mathrm{mM}$ Tris-HCl buffer at $\mathrm{pH} 7.7$, containing $10 \mathrm{mM} \mathrm{NaCl}, 0.1 \mathrm{mM}$ EGTA, $0.5 \mathrm{mM}$ EDTA, $0.5 \mathrm{mM}$ spermidine, $0.15 \mathrm{mM}$ spermine, $0.5 \%$ Tergitol NP-10, $1 \mathrm{mM}$ dithiothreitol, $1 \mathrm{mM}$ phenylmethylsulfonyl fluoride) in a Dounce homogenizer. The homogenates were filtered through two layers of cheesecloth and, following dilution with eight tissue volumes of $2.2 \mathrm{M}$ sucrose in the homogenization buffer, applied to a $10-\mathrm{mL}$ cushion of $2 \mathrm{M}$ sucrose in homogenization buffer and spun at $24,000 \mathrm{rpm}$ for $60 \mathrm{~min}$ at $2^{\circ} \mathrm{C}$ in a prechilled SW28 rotor. The resultant nuclear pellet as well as the supernatant were subjected to RNA extraction. For NIH3T3 and MEFs, cells were lysed in an ice-cold hypotonic lysis buffer $(10 \mathrm{mM}$ Tris- $\mathrm{HCl}$ at $\mathrm{pH} 7.4,10 \mathrm{mM} \mathrm{NaCl}, 3 \mathrm{mM} \mathrm{MgCl}_{2}, 0.3 \%$ NP-40) supplemented with protease inhibitor cocktail (Sigma). After incubation for $5 \mathrm{~min}$ on ice with occasional pipetting, lysates were separated into nuclear and cytoplasmic fractions by centrifuging at $600 \mathrm{~g}$ for $5 \mathrm{~min}$ at $4^{\circ} \mathrm{C}$. RNAs were extracted from each fraction using Trizol reagent (Life Technologies) and subjected to RTqPCR.

Per2AS overexpression and knockdown

A Per2AS variant 2 overexpression plasmid was generated from $5^{\prime} \mathrm{RACE}$ and $3^{\prime} \mathrm{RACE}$ products that were cloned into pBluescript (Agilent), as well as Per2AS qPCR product cloned into pGEM-T (Promega). After combining 5'RACE and qPCR products in pBlueScript using HindIII and XhoI sites, the $3^{\prime}$ RACE product was also inserted using XhoI and KpnI sites. The full length was then transferred to pcDNA3.1-mycHis (Invitrogen), and its sequence was verified by Sanger sequencing. DNA transfection was performed with either FuGENE6 Reagent (Promega) or Lipofectamine 2000 (Life Technologies) using Opti-MEM (Gibco), while gapmers were introduced via gymnosis (Stein et al. 2010) with the final concentration of $100 \mathrm{nM}$ (Supplemental Table S1).

\section{Lentivirus generation and transduction}

For virus production, either control or Per2 shRNA as well as viral packaging vectors were transfected into HEK293/T17 cells according to the manufacturer's instruction (ViraPower Lentiviral Expression System, Life Technologies). Cell culture media was collected after $48 \mathrm{~h}$, ultracentrifuged at $70,000 \mathrm{~g}$ for $2 \mathrm{~h}$ at room temperature, then resuspended in target cell-specific media. Viral media and $20 \mu \mathrm{g} / \mathrm{mL}$ polybrene (Millipore) were added to target cells, and cells were harvested $48-72 \mathrm{~h}$ after viral transduction.

\section{Mathematical model}

Our model of Per2-Per2AS interactions (Battogtokh et al. 2018) was based on an earlier model (Relogio et al. 2011) of the mammalian circadian clock, which did not take Per2AS into account. Our model of the basic negative feedback loop, whereby PER2:CRY inhibits BMAL:CLOCK (without additional feedback loops through REV-ERB and ROR), consisted of 15 ordinary differential equations (ODEs) with 44 parameter values (rate constants, binding constants, etc.). To illustrate some of the results of this detailed model, we present here a simpler model of the negative 
feedback loop (Supplemental Fig. S6A) based on Goodwin's original model (Goodwin 1965), with an important modification suggested by Bliss, Painter, and Marr (Bliss et al. 1982). Per2AS RNA was added to the basic Goodwin model by assuming that Per2AS and Per2 mutually inhibit each other pretranscriptionally. The ODEs describing the model are presented in Supplemental Figure S6B. The model with Per2AS consists of all four ODEs, as written. The model without Per2AS consists of the first three ODEs only, and the blue factor in the first ODE is set $=1$. These two models were simulated with the XPP-AUT program (http://www.math .pitt.edu/ bard/xpp/xpp.html) using the parameter values given in Supplemental Table S2. The parameter values were chosen to give oscillations with a period close to $24 \mathrm{~h}$ and reasonable amplitudes and phase relations of the variables (Supplemental Fig. S6C. In particular, the model with Per2AS was constrained to fit experimental observations in mouse liver that the level of Per2AS is $\sim 5 \%$ of Per2 and that Per2AS and Per2 are expressed antiphasically (Koike et al. 2012). In Supplemental Figure S6D we compare the robustness of oscillations in the models with and without Per2AS, in terms of the range of $a_{1}$ values (the maximum rate of synthesis of Per2 mRNA) over which the models exhibit oscillations. By this measure, the model with Per2AS is considerably more robust than the model without Per2AS. In Supplemental Figure S6E, we show the domains of oscillation for both models on a two-parameter diagram: $a_{1}$ and $b_{3}$, where $b_{3}$ is the maximum rate of degradation of phosphorylated PER2 in the nucleus. This diagram also shows a larger region of oscillations in the model with Per2AS. Even if we restrict our attention to the regions of "circadian" oscillations $(22<$ period $[\mathrm{h}]<26)$ in parameter space, the model with Per2AS (blue lines in Supplemental Fig. S6E) is noticeably more robust than the model without Per2AS (black lines).

\section{Competing interest statement}

The authors declare no competing interests.

\section{Acknowledgment}

We thank Dr. Ueli Schibler (Université de Genève) for providing the Bmal1-luc cell line, Dr. Seung-Hee Yoo (University of Texas Health Science Center at Houston) for PER2::LUC MEFs, and Dr. Andrew Liu (University of Florida) for Per2 shRNA vectors (Ramanathan et al. 2014). We also thank Melissa Makris (Flow Cytometry Resource Laboratory, Department of Biomedical Sciences and Pathobiology, Center for Molecular Medicine and Infectious Diseases, Virginia-Maryland Regional College of Veterinary Medicine, Virginia Polytechnic Institute and State University), Dr. Nicolaas Baudoin and Dr. Daniela Cimini (Department of Biological Sciences, Virginia Polytechnic Institute and State University), and Tsubasa Toda and Akari Ueta (Department of Environmental and Life Sciences, Toyohashi University of Technology) for technical assistance. We also thank all the past an current members of the Green, Takahashi, and Kojima laboratories for invaluable discussion. This work was supported by the Luther and Alice Hamlett Undergraduate Research Support (to C.T.S), Howard Hughes Medical Institute (to J.S.T), and National Institutes of Health GM127122 (to C.B.G), and GM126223 (to S.K.). J.S.T. is an Investigator in the Howard Hughes Medical Institute.

Author contributions: R.A.M., A.N.C., J.C.D., L.P.F., K.L.H., N.M., A.P., C.T.S., L.Z., N.K., J.J.T., and S.K. acquired, analyzed, and interpreted the data. R.A.M., N.K., J.J.T., C.B.G., J.S.T., and S.K., drafted the work and/or critically revised the manuscript for intellectual content. All the authors approved of the manuscript and are accountable for the scientific integrity.

\section{References}

Anderson KM, Anderson DM, McAnally JR, Shelton JM, BasselDuby R, Olson EN. 2016. Transcription of the non-coding RNA upperhand controls Hand2 expression and heart development. Nature 539: 433-436. doi:10.1038/nature20128

Atger F, Gobet C, Marquis J, Martin E, Wang J, Weger B, Lefebvre G, Descombes P, Naef F, Gachon F. 2015. Circadian and feeding rhythms differentially affect rhythmic mRNA transcription and translation in mouse liver. Proc Natl Acad Sci 112: E6579-E6588. doi:10.1073/pnas.1515308112

Bae K, Jin X, Maywood ES, Hastings MH, Reppert SM, Weaver DR. 2001. Differential functions of mPer1, mPer2, and mPer3 in the SCN circadian clock. Neuron 30: 525-536. doi:10.1016/S0896-6273(01)00302-6

Baggs JE, Price TS, DiTacchio L, Panda S, Fitzgerald GA, Hogenesch JB. 2009. Network features of the mammalian circadian clock. PLoS Biol 7: e1000052. doi:10.1371/journal.pbio .1000052

Balsalobre A, Damiola F, Schibler U. 1998. A serum shock induces circadian gene expression in mammalian tissue culture cells. Cell 93: 929-937. doi:10.1016/S0092-8674(00)81199-X

Battogtokh D, Kojima S, Tyson JJ. 2018. Modeling the interactions of sense and antisense period transcripts in the mammalian circadian clock network. PLOS Comput Biol 14: e1005957. doi:10.1371/journal.pcbi.1005957

Bertone P, Stolc V, Royce TE, Rozowsky JS, Urban AE, Zhu X, Rinn JL, Tongprasit W, Samanta M, Weissman S, et al. 2004. Global identification of human transcribed sequences with genome tiling arrays. Science 306: 2242-2246. doi:10.1126/sci ence. 1103388

Bliss RD, Painter PR, Marr AG. 1982. Role of feedback inhibition in stabilizing the classical operon. J Theor Biol 97: 177-193. doi:10.1016/0022-5193/82/90098-4

Bond AM, Vangompel MJ, Sametsky EA, Clark MF, Savage JC, Disterhoft JF, Kohtz JD. 2009. Balanced gene regulation by an embryonic brain ncRNA is critical for adult hippocampal GABA circuitry. Nat Neurosci 12: 1020-1027. doi:10.1038/ nn.2371

Carninci P, Kasukawa T, Katayama S, Gough J, Frith MC, Maeda N, Oyama R, Ravasi T, Lenhard B, Wells C, et al. 2005. The transcriptional landscape of the mammalian genome. Science 309: 1559-1563. doi:10.1126/science.1112014

Carthew RW, Sontheimer EJ. 2009. Origins and mechanisms of miRNAs and siRNAs. Cell 136: 642-655. doi:10.1016/j.cell .2009 .01 .035

Chen LL. 2016. Linking long noncoding RNA localization and function. Trends Biochem Sci 41: 761-772. doi:10.1016/j tibs.2016.07.003

Chen R, Schirmer A, Lee Y, Lee H, Kumar V, Yoo SH, Takahashi JS, Lee C. 2009. Rhythmic PER abundance defines a critical nodal point for negative feedback within the circadian clock mechanism. Mol Cell 36: 417-430. doi:10.1016/j.molcel .2009 .10 .012

Clemson CM, Hutchinson JN, Sara SA, Ensminger AW, Fox AH, Chess A, Lawrence JB. 2009. An architectural role for a nuclear noncoding RNA: NEAT1 RNA is essential for the structure of paraspeckles. Mol Cell 33: 717-726. doi:10.1016/j.molcel .2009 .01 .026

Davis CA, Hitz BC, Sloan CA, Chan ET, Davidson JM, Gabdank I, Hilton JA, Jain K, Baymuradov UK, Narayanan AK, et al. 2018. 
The Encyclopedia of DNA Elements (ENCODE): data portal update. Nucleic Acids Res 46: D794-D801. doi:10.1093/nar/ gkx1081

Derrien T, Johnson R, Bussotti G, Tanzer A, Djebali S, Tilgner H, Guernec G, Martin D, Merkel A, Knowles DG, et al. 2012. The GENCODE v7 catalog of human long noncoding RNAs: analysis of their gene structure, evolution, and expression. Genome Res 22: 1775-1789. doi:10.1101/gr.132159.111

Dinger ME, Amaral PP, Mercer TR, Pang KC, Bruce SJ, Gardiner BB, Askarian-Amiri ME, Ru K, Solda G, Simons C, et al. 2008. Long noncoding RNAs in mouse embryonic stem cell pluripotency and differentiation. Genome Res 18: 1433-1445. doi:10 $.1101 /$ gr.078378.108

Djebali S, Davis CA, Merkel A, Dobin A, Lassmann T, Mortazavi A, Tanzer A, Lagarde J, Lin W, Schlesinger F, et al. 2012. Landscape of transcription in human cells. Nature 489: 101-108. doi:10.1038/nature11233

The ENCODE Project Consortium. 2012. An integrated encyclopedia of DNA elements in the human genome. Nature 489: 57-74. doi:10.1038/nature11247

Engreitz JM, Haines JE, Perez EM, Munson G, Chen J, Kane M, McDonel PE, Guttman M, Lander ES. 2016. Local regulation of gene expression by lncRNA promoters, transcription and splicing. Nature 539: 452-455. doi:10.1038/nature20149

Faghihi MA, Wahlestedt C. 2009. Regulatory roles of natural antisense transcripts. Nat Rev Mol Cell Biol 10: 637-643. doi:10 $.1038 / \mathrm{nrm} 2738$

Fang B, Everett LJ, Jager J, Briggs E, Armour SM, Feng D, Roy A, Gerhart-Hines Z, Sun Z, Lazar MA. 2014. Circadian enhancers coordinate multiple phases of rhythmic gene transcription in vivo. Cell 159: 1140-1152. doi:10.1016/j.cell.2014.10.022

Feng J, Bi C, Clark BS, Mady R, Shah P, Kohtz JD. 2006. The Evf-2 noncoding RNA is transcribed from the Dlx-5/6 ultraconserved region and functions as a Dlx-2 transcriptional coactivator. Genes Dev 20: 1470-1484. doi:10.1101/gad.1416106

Goodman AJ, Daugharthy ER, Kim J. 2013. Pervasive antisense transcription is evolutionarily conserved in budding yeast. Mol Biol Evol 30: 409-421. doi:10.1093/molbev/mss240

Goodwin BC. 1965. Oscillatory behavior in enzymatic control processes. Adv Enzyme Regul 3: 425-437. doi:10.1016/00652571(65)90067-1

Groff AF, Sanchez-Gomez DB, Soruco MML, Gerhardinger C, Barutcu AR, Li E, Elcavage L, Plana O, Sanchez LV, Lee JC, et al. 2016. In vivo characterization of Linc-p21 reveals functional cis-regulatory DNA elements. Cell Rep 16: 21782186. doi:10.1016/j.celrep.2016.07.050

Guttman M, Amit I, Garber M, French C, Lin MF, Feldser D, Huarte M, Zuk O, Carey BW, Cassady JP, et al. 2009. Chromatin signature reveals over a thousand highly conserved large non-coding RNAs in mammals. Nature 458: 223-227. doi:10 .1038 /nature07672

Halley P, Kadakkuzha BM, Faghihi MA, Magistri M, Zeier Z, Khorkova O, Coito C, Hsiao J, Lawrence M, Wahlestedt C. 2014. Regulation of the apolipoprotein gene cluster by a long noncoding RNA. Cell Rep 6: 222-230. doi:10.1016/j.celrep .2013 .12 .015

He Y, Vogelstein B, Velculescu VE, Papadopoulos N, Kinzler KW. 2008. The antisense transcriptomes of human cells. Science 322: 1855-1857. doi:10.1126/science.1163853

He B, Nohara K, Park N, Park YS, Guillory B, Zhao Z, Garcia JM, Koike N, Lee CC, Takahashi JS, et al. 2016. The small molecule Nobiletin targets the molecular oscillator to enhance circadian rhythms and protect against metabolic syndrome. Cell Metab 23: 610-621. doi:10.1016/j.cmet.2016.03.007
Janich P, Arpat AB, Castelo-Szekely V, Lopes M, Gatfield D. 2015. Ribosome profiling reveals the rhythmic liver translatome and circadian clock regulation by upstream open reading frames. Genome Res 25: 1848-1859. doi:10.1101/gr.195404 .115

Johnsson P, Lipovich L, Grandér D, Morris KV. 2014. Evolutionary conservation of long non-coding RNAs; sequence, structure, function. Biochim Biophys Acta 1840: 1063-1071. doi:10.1016/j.bbagen.2013.10.035

Kaikkonen MU, Adelman K. 2018. Emerging roles of non-coding RNA transcription. Trends Biochem Sci 43: 654-667. doi:10 $.1016 / j$. tibs.2018.06.002

Katayama S, Tomaru Y, Kasukawa T, Waki K, Nakanishi M, Nakamura M, Nishida H, Yap CC, Suzuki M, Kawai J, et al. 2005. Antisense transcription in the mammalian transcriptome. Science 309: 1564-1566. doi:10.1126/science.1112009

Khaitovich P, Kelso J, Franz H, Visagie J, Giger T, Joerchel S, Petzold E, Green RE, Lachmann M, Pääbo S. 2006. Functionality of intergenic transcription: an evolutionary comparison. PLOS Genet 2: e171. doi:10.1371/journal.pgen.0020171

Khorkova O, Myers AJ, Hsiao J, Wahlestedt C. 2014. Natural antisense transcripts. Hum Mol Genet 23: R54-R63. doi:10 $.1093 / \mathrm{hmg} / \mathrm{ddu} 207$

Koike N, Yoo SH, Huang HC, Kumar V, Lee C, Kim TK, Takahashi JS. 2012. Transcriptional architecture and chromatin landscape of the core circadian clock in mammals. Science 338: 349-354. doi:10.1126/science.1226339

Kojima S, Gatfield D, Esau CC, Green CB. 2010. MicroRNA-122 modulates the rhythmic expression profile of the circadian deadenylase Nocturnin in mouse liver. PLoS One 5: e11264. doi:10.1371/journal.pone.0011264

Kramer C, Loros JJ, Dunlap JC, Crosthwaite SK. 2003. Role for antisense RNA in regulating circadian clock function in Neurospora crassa. Nature 421: 948-952. doi:10.1038/nature01427

Lee JS, Mendell JT. 2020. Antisense-mediated transcript knockdown triggers premature transcription termination. Mol Cell 77: 1044-1054.e3. doi:10.1016/j.molcel.2019.12.011

Lee JT, Davidow LS, Warshawsky D. 1999. Tsix, a gene antisense to Xist at the X-inactivation centre. Nat Genet 21: 400-404. doi:10.1038/7734

Lee JE, Bennett CF, Cooper TA. 2012. RNase H-mediated degradation of toxic RNA in myotonic dystrophy type 1. Proc Natl Acad Sci 109: 4221-4226. doi:10.1073/pnas.1117019109

Littleton ES, Childress ML, Gosting ML, Jackson AN, Kojima S. 2020. Genome-wide correlation analysis to identify amplitude regulators of circadian transcriptome output. Sci Rep 10: 21839. doi:10.1038/s41598-020-78851-9

Liu AC, Welsh DK, Ko CH, Tran HG, Zhang EE, Priest AA, Buhr ED, Singer O, Meeker K, Verma IM, et al. 2007. Intercellular coupling confers robustness against mutations in the SCN circadian clock network. Cell 129: 605-616. doi:10.1016/j.cell 2007.02.047

Lowrey PL, Takahashi JS. 2004. Mammalian circadian biology: elucidating genome-wide levels of temporal organization. Annu Rev Genomics Hum Genet 5: 407-441. doi:10.1146/ annurev.genom.5.061903.175925

Luck S, Thurley K, Thaben PF, Westermark PO. 2014. Rhythmic degradation explains and unifies circadian transcriptome and proteome data. Cell Rep 9: 741-751. doi:10.1016/j.celrep.2014 .09 .021

Marchese FP, Raimondi I, Huarte M. 2017. The multidimensional mechanisms of long noncoding RNA function. Genome Biol 18: 206. doi:10.1186/s13059-017-1348-2 
Menet JS, Rodriguez J, Abruzzi KC, Rosbash M. 2012. NascentSeq reveals novel features of mouse circadian transcriptional regulation. Elife 1: e00011. doi:10.7554/eLife.00011

Mercer TR, Dinger ME, Mattick JS. 2009. Long non-coding RNAs: insights into functions. Nat Rev Genet 10: 155-159. doi:10.1038/nrg2521

Modarresi F, Faghihi MA, Lopez-Toledano MA, Fatemi RP, Magistri M, Brothers SP, van der Brug MP, Wahlestedt C. 2012. Inhibition of natural antisense transcripts in vivo results in gene-specific transcriptional upregulation. Nat Biotechnol 30: 453-459. doi:10.1038/nbt.2158

Morf J, Rey G, Schneider K, Stratmann M, Fujita J, Naef F, Schibler U. 2012. Cold-inducible RNA-binding protein modulates circadian gene expression posttranscriptionally. Science 338: 379-383. doi:10.1126/science.1217726

Morris KV, Santoso S, Turner AM, Pastori C, Hawkins PG. 2008. Bidirectional transcription directs both transcriptional gene activation and suppression in human cells. PLoS Genet 4: e1000258. doi:10.1371/journal.pgen.1000258

Nicholson JM, Macedo JC, Mattingly AJ, Wangsa D, Camps J, Lima V, Gomes AM, Dória S, Ried T, Logarinho E, et al. 2015. Chromosome mis-segregation and cytokinesis failure in trisomic human cells. Elife 4: e05068. doi:10.7554/eLife .05068

Oklejewicz M, Destici E, Tamanini F, Hut RA, Janssens R, van der Horst GT. 2008. Phase resetting of the mammalian circadian clock by DNA damage. Curr Biol 18: 286-291. doi:10 .1016/j.cub.2008.01.047

Panda AC, Grammatikakis I, Munk R, Gorospe M, Abdelmohsen K. 2017. Emerging roles and context of circular RNAs. Wiley Interdiscip Rev RNA 8: e1386. doi:10.1002/wrna.1386

Pang KC, Frith MC, Mattick JS. 2006. Rapid evolution of noncoding RNAs: lack of conservation does not mean lack of function. Trends Genet 22: 1-5. doi:10.1016/j.tig.2005.10.003

Pendergast JS, Friday RC, Yamazaki S. 2010. Distinct functions of Period 2 and Period 3 in the mouse circadian system revealed by in vitro analysis. PLoS One 5: e8552. doi:10.1371/journal .pone.0008552

Phatnani HP, Greenleaf AL. 2006. Phosphorylation and functions of the RNA polymerase II CTD. Genes Dev 20: 2922-2936. doi:10.1101/gad.1477006

Ponjavic J, Ponting CP, Lunter G. 2007. Functionality or transcriptional noise? Evidence for selection within long noncoding RNAs. Genome Res 17: 556-565. doi:10.1101/gr.6036807

Ramanathan C, Xu H, Khan SK, Shen Y, Gitis PJ, Welsh DK, Hogenesch JB, Liu AC. 2014. Cell type-specific functions of period genes revealed by novel adipocyte and hepatocyte circadian clock models. PLoS Genet 10: e1004244. doi:10.1371/ journal.pgen.1004244

Ran FA, Hsu PD, Wright J, Agarwala V, Scott DA, Zhang F. 2013. Genome engineering using the CRISPR-Cas9 system. Nat Protoc 8: 2281-2308. doi:10.1038/nprot.2013.143

Relogio A, Westermark PO, Wallach T, Schellenberg K, Kramer A, Herzel H. 2011. Tuning the mammalian circadian clock: robust synergy of two loops. PLoS Comput Biol 7: e1002309. doi:10.1371/journal.pcbi.1002309

Rhind N, Chen Z, Yassour M, Thompson DA, Haas BJ, Habib N, Wapinski I, Roy S, Lin MF, Heiman DI, et al. 2011. Comparative functional genomics of the fission yeasts. Science 332: 930-936. doi:10.1126/science.1203357

Røsok O, Sioud M. 2004. Systematic identification of sense-antisense transcripts in mammalian cells. Nat Biotechnol 22: 104-108. doi:10.1038/nbt925

Sato TK, Panda S, Miraglia LJ, Reyes TM, Rudic RD, McNamara P, Naik KA, FitzGerald GA, Kay SA, Hogenesch JB. 2004. A functional genomics strategy reveals Rora as a component of the mammalian circadian clock. Neuron 43: 527-537. doi:10 .1016/j.neuron.2004.07.018

Sauman I, Reppert SM. 1996. Circadian clock neurons in the silkmoth Antheraea pernyi: novel mechanisms of Period protein regulation. Neuron 17: 889-900. doi:10.1016/S0896-6273(00) 80220-2

Schmutz I, Ripperger JA, Baeriswyl-Aebischer S, Albrecht U. 2010. The mammalian clock component PERIOD2 coordinates circadian output by interaction with nuclear receptors. Genes Dev 24: 345-357. doi:10.1101/gad.564110

Shearman LP, Sriram S, Weaver DR, Maywood ES, Chaves I, Zheng B, Kume K, Lee CC, van der Horst GT, Hastings MH, et al. 2000. Interacting molecular loops in the mammalian circadian clock. Science 288: 1013-1019. doi:10.1126/science .288 .5468 .1013

Sleutels F, Zwart R, Barlow DP. 2002. The non-coding Air RNA is required for silencing autosomal imprinted genes. Nature 415: 810-813. doi:10.1038/415810a

Smilinich NJ, Day CD, Fitzpatrick GV, Caldwell GM, Lossie AC, Cooper PR, Smallwood AC, Joyce JA, Schofield PN, Reik W, et al. 1999. A maternally methylated CpG island in KvLQT1 is associated with an antisense paternal transcript and loss of imprinting in Beckwith-Wiedemann syndrome. Proc Natl Acad Sci 96: 8064-8069. doi:10.1073/pnas.96.14.8064

Sopher BL, Ladd PD, Pineda VV, Libby RT, Sunkin SM, Hurley JB, Thienes CP, Gaasterland T, Filippova GN, La Spada AR. 2011. CTCF regulates ataxin-7 expression through promotion of a convergently transcribed, antisense noncoding RNA. Neuron 70: 1071-1084. doi:10.1016/j.neuron.2011.05.027

Stein CA, Hansen JB, Lai J, Wu S, Voskresenskiy A, HØg A, Worm J, Hedtjärn M, Souleimanian N, Miller P, et al. 2010. Efficient gene silencing by delivery of locked nucleic acid antisense oligonucleotides, unassisted by transfection reagents. Nucleic Acids Res 38: e3. doi:10.1093/nar/gkp841

Sun M, Hurst LD, Carmichael GG, Chen J. 2006. Evidence for variation in abundance of antisense transcripts between multicellular animals but no relationship between antisense transcriptionand organismic complexity. Genome Res 16: 922-933. doi:10.1101/gr.5210006

Takahashi JS. 2017. Transcriptional architecture of the mammalian circadian clock. Nat Rev Genet 18: 164-179. doi:10.1038/ nrg.2016.150

Takahashi JS, Hong HK, Ko CH, McDearmon EL. 2008. The genetics of mammalian circadian order and disorder: implications for physiology and disease. Nat Rev Genet 9: 764-775. doi: $10.1038 / \mathrm{nrg} 2430$

Tamiya H, Ogawa S, Ouchi Y, Akishita M. 2016. Rigid cooperation of Per1 and Per2 proteins. Sci Rep 6: 32769. doi:10 $.1038 /$ srep32769

Villegas VE, Rahman MF, Fernandez-Barrena MG, Diao Y, Liapi E, Sonkoly E, Ståhle M, Pivarcsi A, Annaratone L, Sapino A, et al. 2014. Identification of novel non-coding RNA-based negative feedback regulating the expression of the oncogenic transcription factor GLI1. Mol Oncol 8: 912-926. doi:10.1016/j .molonc.2014.03.009

Vitaterna $\mathrm{MH}$, Ko $\mathrm{CH}$, Chang $\mathrm{AM}$, Buhr ED, Fruechte EM, Schook A, Antoch MP, Turek FW, Takahashi JS. 2006. The mouse Clock mutation reduces circadian pacemaker amplitude and enhances efficacy of resetting stimuli and phase-response curve amplitude. Proc Nat1 Acad Sci 103: 9327-9332. doi:10.1073/pnas.0603601103

Vollmers C, Schmitz RJ, Nathanson J, Yeo G, Ecker JR, Panda S. 2012. Circadian oscillations of protein-coding and regulatory 
RNAs in a highly dynamic mammalian liver epigenome. Cell Metab 16: 833-845. doi:10.1016/j.cmet.2012.11.004

Wanowska E, Kubiak MR, Rosikiewicz W, Makałowska I, Szcześniak MW. 2018. Natural antisense transcripts in diseases: from modes of action to targeted therapies. Wiley Interdiscip Rev RNA 9: e1461. doi:10.1002/wrna.1461

Wight M, Werner A. 2013. The functions of natural antisense transcripts. Essays Biochem 54: 91-101. doi:10.1042/ bse0540091

Xu Z, Wei W, Gagneur J, Perocchi F, Clauder-Münster S, Camblong J, Guffanti E, Stutz F, Huber W, Steinmetz LM. 2009. Bidirectional promoters generate pervasive transcription in yeast. Nature 457: 1033-1037. doi:10.1038/nature07728

Xu Z, Wei W, Gagneur J, Clauder-Münster S, Smolik M, Huber W, Steinmetz LM. 2011. Antisense expression increases gene expression variability and locus interdependency. Mol Syst Biol 7: 468. doi:10.1038/msb.2011.1

Xue Z, Ye Q, Anson SR, Yang J, Xiao G, Kowbel D, Glass NL, Crosthwaite SK, Liu Y. 2014. Transcriptional interference by antisense RNA is required for circadian clock function. $\mathrm{Na}$ ture 514: 650-653. doi:10.1038/nature 13671

Yassour M, Pfiffner I, Levin JZ, Adiconis X, Gnirke A, Nusbaum C, Thompson DA, Friedman N, Regev A. 2010. Strand-specific RNA sequencing reveals extensive regulated long antisense transcripts that are conserved across yeast species. Genome Biol 11: R87. doi:10.1186/gb-2010-11-8-r87

Yoo SH, Yamazaki S, Lowrey PL, Shimomura K, Ko CH, Buhr ED, Siepka SM, Hong HK, Oh WJ, Yoo OJ, et al. 2004. PERIOD2::
LUCIFERASE real-time reporting of circadian dynamics reveals persistent circadian oscillations in mouse peripheral tissues. Proc Natl Acad Sci 101: 5339-5346. doi:10.1073/pnas .0308709101

Yoo SH, Kojima S, Shimomura K, Koike N, Buhr ED, Furukawa T, Ko CH, Gloston G, Ayoub C, Nohara K, et al. 2017. Period2 3'UTR and microRNA-24 regulate circadian rhythms by repressing PERIOD2 protein accumulation. Proc Natl Acad Sci 114: E8855-E8864. doi:10.1073/pnas.1706611114

Zhang EE, Liu AC, Hirota T, Miraglia LJ, Welch G, Pongsawakul PY, Liu X, Atwood A, Huss JW, Janes J, et al. 2009. A genomewide RNAi screen for modifiers of the circadian clock in human cells. Cell 139: 199-210. doi:10.1016/j.cell.2009.08.031

Zhang R, Lahens NF, Ballance HI, Hughes ME, Hogenesch JB. 2014. A circadian gene expression atlas in mammals: implications for biology and medicine. Proc Natl Acad Sci 111: 16219-16224. doi:10.1073/pnas.1408886111

Zhao J, Sun BK, Erwin JA, Song JJ, Lee JT. 2008. Polycomb proteins targeted by a short repeat RNA to the mouse X chromosome. Science 322: 750-756. doi:10.1126/science.1163045

Zhao X, Hirota T, Han X, Cho H, Chong LW, Lamia K, Liu S, Atkins AR, Banayo E, Liddle C, et al. 2016. Circadian amplitude regulation via FBXW7-targeted REV-ERBa degradation. Cell 165: 1644-1657. doi:10.1016/j.cell.2016.05.012

Zheng B, Larkin DW, Albrecht U, Sun ZS, Sage M, Eichele G, Lee CC, Bradley A. 1999. The mPer2 gene encodes a functional component of the mammalian circadian clock. Nature 400: 169-173. doi:10.1038/22118 


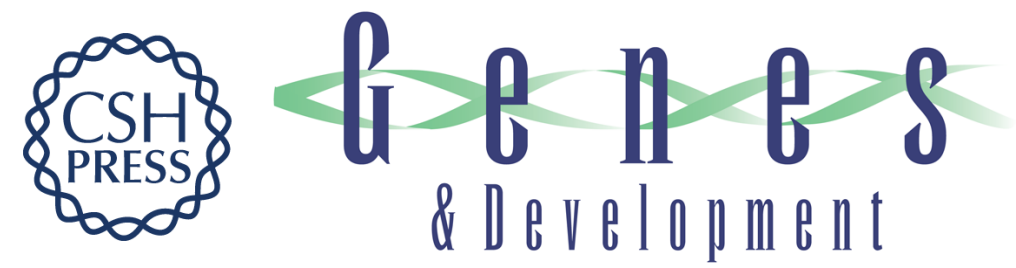

\section{Natural antisense transcript of Period2, Per2AS, regulates the amplitude of the mouse circadian clock}

Rebecca A. Mosig, Allison N. Castaneda, Jacob C. Deslauriers, et al.

Genes Dev. 2021, 35: originally published online May 20, 2021

Access the most recent version at doi:10.1101/gad.343541.120

\section{Supplemental http://genesdev.cshlp.org/content/suppl/2021/05/17/gad.343541.120.DC1 Material}

References This article cites 92 articles, 26 of which can be accessed free at: http://genesdev.cshlp.org/content/35/11-12/899.full.html\#ref-list-1

Creative This article is distributed exclusively by Cold Spring Harbor Laboratory Press for the first Commons six months after the full-issue publication date (see

License http://genesdev.cshlp.org/site/misc/terms.xhtml). After six months, it is available under a Creative Commons License (Attribution-NonCommercial 4.0 International), as described at http://creativecommons.org/licenses/by-nc/4.0/.

Email Alerting Receive free email alerts when new articles cite this article - sign up in the box at the top Service right corner of the article or click here. 